You Said You Would! The Predictability of Other's Behavior From Their Intentions Determines Predictive Biases in Action Perception.

Hudson, Matthew

http://hdl.handle.net/10026.1/9560

10.1037/xhp0000451

Journal of Experimental Psychology: Human Perception and Performance

American Psychological Association (APA)

All content in PEARL is protected by copyright law. Author manuscripts are made available in accordance with publisher policies. Please cite only the published version using the details provided on the item record or document. In the absence of an open licence (e.g. Creative Commons), permissions for further reuse of content should be sought from the publisher or author. 


\title{
You Said You Would! The Predictability of Other's Behavior from their Intentions Determines Predictive Biases in Action Perception.
}

Matthew Hudson*, Patric Bach, and Toby Nicholson.

School of Psychology, University of Plymouth, UK

\author{
Author Note
}

Matthew Hudson now affiliated with University of Turku, Turku, Finland. Toby Nicholson now affiliated with University of Kent, Canterbury, UK.

This work was supported by the Economic and Social Research Council [grant number ES/J019178/1] awarded to Patric Bach.

Correspondence: Matthew Hudson, University of Turku, Turku PET Centre, Turku University Hospital, Kiinamyllynkatu 4-8, rak.14, FI-20520 Turku, Finalnd. Tel: +358 (0) 23132786, Email: matthew.hudson@utu.fi 


\begin{abstract}
The perception of an action is shifted farther along the observed trajectory if the observer has prior knowledge of the actor's intention. This intention-action prediction effect is explained by predictive perception models, wherein sensory input is interpreted in light of expectancies. This study altered the precision of the prediction by varying the predictability of the action from the intention, to increase/decrease the predictive perceptual bias. Participants heard an actor state an intention ('I'll take it"/"I'll leave it") before the actor reached/withdrew from an object, thus confirming or contradicting the intention. The intention was predictive of the action ( $75 \%$ congruency) for one group and counter-predictive (25\%) for another. The action disappeared mid-movement and participants estimated the disappearance position. The intention-action prediction effect was greater if the intention was predictive than if counter-predictive. However, participants needed to explicitly know the predictability rates (Experiments 1 and 3). No group differences emerged when both groups believed the intention was non-predictive (Experiment 2a), nor when a non-predictive intention was believed to be (counter)predictive (Experiment $2 b$ ). The perception of others behavior is determined by its predictability from their intentions, and the precision of our social predictions is adapted to individual differences in behavior.
\end{abstract}

Keywords: representational momentum; action prediction; predictive coding; implicit theory of mind; intentions; action understanding; perceptual prediction; action perception; awareness, associative learning. 


\section{Public Significance Statement}

Our prior knowledge of others mental states enables us to see other people's behaviour not as simply the motion of their limbs, but in terms of the thoughts, emotions, and intentions that guide them. For example, we have previously demonstrated that the perception of someone reaching towards an object is distorted to appear as nearer the object than it actually is if the actor had previously stated an intention of "I'll take it" than if he had stated the opposite (e.g. "I'll leave it"). We show that the strength of this perceptual distortion is larger if the actor's intention is highly predictive of the action than if the actor is unlikely to do as they had said. Our perception of others behaviour is shaped by what we expect them to do, and how strong these predictions are, allowing us to adapt to different individuals and different situations. 


\section{You Said You Would! The Predictability of Other's Behavior from their Intentions Determines Predictive Biases in Action Perception.}

The ability to pre-emptively adapt our behavior by predicting changes in the environment is crucial for interacting with a dynamic world. Nowhere is this more necessary than in social interactions, where anticipating the behavior of potential mates, alliances, and competitors contributes to well-being and survival. However, predictions in the social domain are all the more challenging as the goals and intentions that cause others' behavior are opaque (Dennett, 1987; Saxe, Tenenbaum, \& Carey, 2005). For this reason, humans are exquisitely sensitive to cues that convey what others are thinking and the possible actions they may make, from the objects nearby (e.g., Bach, Knoblich, Gunter, Friederici \& Prinz, 2005; for reviews see Bach, Nicholson \& Hudson, 2014; van Elk, van Schie, \& Bekkering, 2014), where they are looking (Joyce, Schenke \& Bach, 2016; Teufel, Fletcher, \& Davis, 2010), or what they are saying (e.g., Hudson, Nicholson, Ellis \& Bach, 2016).

Recently, it has been argued that the ability to make such predictions is at least to some extent perceptual (Bach, Nicholson \& Hudson, 2014; Bach \& Schenke, in press; Csibra, 2007; Kilner, Friston \& Frith, 2007 ${ }^{\mathrm{ab}}$; Zaki, 2013). For example, the perceived position of a moving object is often displaced further along its trajectory, as if shifted forward in time to reflect where it will be in the immediate future. The representational momentum effect denotes the finding that after seeing a moving object suddenly disappear, people report its final position further along its trajectory than it really was, as if its perceptual representation was enriched by predictions of its future course (Freyd \& Fink, 1984; Hubbard, 2005; 2014; flash lag effect: Hubbard, 2013; Nijhawan, 2002). These perceptual distortions show how predictive processes exploit the spatio-temporal regularities of movement (see Goldreich, 2007; Sarrazin, Giraudo, Pailhous \& Bootsma, 2004 for further examples) to maintain a coherent impression of motion even under impoverished viewing 
conditions (Kourtzi \& Shiffrar, 1997; Munger \& Minchew, 2002; for review, see Bach, Hudson \& Nicholson, in prep).

Recent models explain these findings by conceptualizing perception as an inferential process, whereby sensory information is interpreted in light of prior expectations (Bubic, von Cramon \& Schubotz, 2010; Clark, 2013; den Ouden, Kok, \& De Lange, 2012; Friston \& Kiebel, 2009; Markov \& Kennedy, 2013). The feed forward stream of incoming sensory information is weighted according to predictions from higher cortical levels, reflecting prior knowledge of the world, such as how objects typically move in the example of motion perception. This enables correctly predicted stimuli that already confirm what we know of the world - a continuation along the current trajectory - to be identified with minimal processing and ambiguous input to be perceived in light of these expectations. In contrast, cognitive resources are devoted to unexpected stimuli that reveal errors in one's model of the world - e.g. a sudden change in direction - which are sent upstream to modify one's predictions and responses. Next to the case of motion perception, there are now more and more examples of top-down information influences on perceptual processing, spanning from judgments of color (Lafer-Sousa, Hermann, \& Conway, 2015), to whether a surface is seen as convex or concave (Stone, Kerrigan, \& Porrill, 2009), or the direction an object is rotating based on one's own actions (Wohlschläger, 2000).

Most recently, models of top-down predictive mechanisms have been brought to bear on social perception (Brown \& Brune, 2012; Csibra, 2007; Hudson et al, 2016; Hudson, Nicholson, Simpson, Ellis \& Bach, 2016; Keysers \& Gazzola, 2014; Kilner, 2011; Kilner, Friston \& Frith, $2007^{\text {ab }}$; Koster-Hale \& Saxe, 2013), where there is similar evidence for perceptual biases. For example, the perceived kinematics of an action are shaped by one's expectations about the complex articulations of human motion (Chatterjee, Freyd, \& Shiffrar, 1996; Kourtzi \& Shiffrar, 1999; Shiffrar \& Freyd, 1990; 1993; Shiffrar, Lichtey, \& Chatterjee, 1997; Orgs, Bestmann, Schuur, \& Haggard, 2011), and the perception of moving body parts is displaced forward in time to a position 
that would've occurred had the action continued (Graf et al., 2007; Hudson, Burnett, \& Jellema, 2012; Hudson, Hong-Liu, \& Jellema, 2009; Hudson \& Jellema, 2011; Jarraya, Amorim, \& Bardy, 2005; Kessler, Gordon, Cessford, \& Lages, 2010; Thornton \& Hayes, 2004; Wilson, Lancaster \& Emmorey, 2010). This enables observers to simulate the kinematics of the action when occluded from view (Parkinson, Springer \& Prinz, 2012), or to predict how and when a person will react to the behavior of others (Manera, Schouten, Verfaillie, \& Becchio, 2013; Neri, Luu, \& Levi, 2006).

These findings suggest that the perception of biological motion may be subject to similar predictive influences as non-biological motion, integrating not only the bottom-up sensory input, but also prior expectations about how humans move. However, what sets human actions apart is that they are intentional and purposeful, self-generated by one's beliefs and desires to achieve distal changes in the environment. A key prediction of top-down models of human social perception is therefore that the perception of others' actions should also directly integrate such inferences about the actors' goals (Bach et al., 2014; Csibra, 2007; Kilner, 2011; Kilner, Friston \& Frith, $2007^{\mathrm{ab}}$ ). Using a representational momentum task, we have shown exactly that (Hudson et al, 2016 ${ }^{\mathrm{ab}}$ ). Participants observed an actor either reach for an object or withdraw from it. The action disappeared midway through its trajectory and participants estimated its disappearance point, providing a measure of its perceived position. Prior to action onset, the observer heard the actor state an intention of either "I'll take it" or "I'll leave it", enabling them to predict the action. For both actions, the perceived position was shifted further along the observed trajectory than it actually was, confirming the classic predictive perceptual bias effect. That is, based on the extrapolation of prior motion, a reach was perceived as nearer the object, and a withdrawal further away. Importantly, however, these predictive biases were influenced by the prior intentional statement. A hand was reported to have disappeared even closer to the goal object when the actor had previously stated that they would take it, and further away when stating they would leave it. 
These results show, first, that an action's perceptual representation is predictively biased, and, second, that these biases result not only from an extrapolation of the prior motion, but that, for the case of human intentional action, the assumed goals of the actor are factored in as well. The data therefore provide first evidence for a mechanism that relies on information about others' goals and translates them into low-level descriptions of the actions they would do as a consequence, which then directly influences the perceptual representation of forthcoming action. Intriguingly, this was the case even though the intention statement was completely non-predictive of the action that would follow. The actor was just as likely to withdraw from the object after saying "I'll take it" than they were to reach towards it, and vice versa. The prediction generated from the intention was therefore not derived from the experimental context itself but drawn from a life time of experience of associating each action with its respective intention. Yet, people and situations differ in how strongly their actions are related to the cues they exhibit. It is therefore crucial that one's internal models of how certain cues predict behavior are constantly adjusted to new situations and individuals. Indeed, in predictive coding models, it is assumed that people can up- and downregulate the weighting given to each predictive cue depending on their reliability in predicting future events, such that cues that are reliably associated with other events shape perception more strongly than less related cues (e.g., Friston \& Kiebel, 2009; Hohwy, 2013).

Here, we tested whether the intention-action prediction effects reported by Hudson et al., $\left(2016^{\mathrm{ab}}\right)$ are subject to the same dynamic adjustments based on the reliability with which a cue predicts forthcoming actions. That is, the same verbal cue ("I'll take it" or "I'll leave it") should affect perceptual action judgments differently, depending on whether the relationship between cue and subsequent action is predictive and therefore supports the prior relationships established by real world experience, or whether it is counter-predictive and therefore undermines the reliability with which these cues predict subsequent behavior. Importantly, as in our prior studies (Hudson, et al., $\left.2016^{\mathrm{ab}}\right)$, the actor's intention statements - and their relationship to their subsequent actions - were 
completely irrelevant to the participants' task of judging the hand's disappearance points. This allows us to test, not only whether inferred intentions automatically affect perceptual action judgments, but also delineate the conditions under which the statements' relationship to subsequent behavior is monitored and, if required, updated.

Showing such effects is crucial to test the central assumption of predictive coding models of social perception that predictive relationships and the weight of different cues in predicting subsequent stimuli are adjusted in light of new information. In addition, such findings would argue against one important alternative explanation of the previous effects. One possibility is that our previous findings of verbal intention statements affecting action perception do not reflect predictive processes per se, but "embodied" effects of word meaning on visual perception. It has long been known, for example, that hearing certain words affects motion perception, such that the word "rise" causes one to perceive upwards motion more effectively and vice versa for "fall", simply due to the embodied knowledge people associate with these words (Dils \& Boroditsky, 2010; Meteyard, Bahrami, \& Vigliocco, 2007). In contrast, if the effects on action perception do not reflect such stable word meaning but the causal role of intentional cues for predicting behavior, then they should be dynamically adjusted to reflect the reliability of these cues.

\section{Experiment 1}

Experiment 1 tested whether the validity with which an actor's intention predicts their forthcoming behavior influences the predictive bias in the perception of their actions. We presented two groups of participants with the modified representational momentum paradigm devised by Hudson et al $\left(2016^{\mathrm{a}}\right)$. After hearing the actor state an intention of either "I'll take it" or "I'll leave it", they observed the actor either reach for an object or withdraw from it. They then judged the hand's sudden disappearance point relative to probe stimuli presented immediately afterwards, which showed the hand in either the same position, or slightly displaced in the direction of motion, 
or in the opposite direction of motion. The crucial manipulation was that for one participant group, the stated intention was highly predictive of the action (correct on $75 \%$ of trials) and therefore supported the previously established relationships between people's intention statements and their subsequent actions. For the second group, the intention was counter-predictive of the action (correct on $25 \%$ of trials), such that the actor typically did the opposite of what he said. The incongruence of prior associations and experimental manipulation in the latter group should lead to a de-weighting of this cue for predictions. The simple hypothesis is that the larger predictive perceptual bias observed for congruent actions than incongruent actions (the intention-action prediction effect) should be greater for the Predictive group, where the intention is predictive of the action, than for the Counter-predictive group, where the intention is counter-predictive of the action.

To maximize the potential of observing an effect in this first experiment, participants were made explicitly aware of how likely the intention was to correctly predict the action. The Predictive group was told that the actor will most likely do as they had said, and the Counter-predictive group was told that the actor will most likely do the opposite of what they had said.

\section{Method}

Participants. Seventy eight participants took part in Experiment 1 (mean age $=21.8$ years, $S D=6.7,67$ females). Participants were randomly assigned to either the Predictive group $(n=40)$ or the Counter-predictive group $(n=38)$. The groups did not differ in terms of age $(t(76)=.022, p=$ $.982)$ or gender composition $\left(X^{2}{ }_{1}=.055, p=.815\right)$. Participants were recruited from Plymouth University, UK and wider community and gave written informed consent prior to taking part. All were right handed, had normal/corrected vision, and spoke English as a native language. They were given either course credit or $£ 6$ for taking part. The experiment was approved by the ethics committee of Plymouth University, UK in agreement with APA and ESRC guidelines and the Declaration of Helsinki. 
Apparatus. The experiment was administered using Presentation (NeuroBS) on a Viglen DQ67SW computer with a Philips Brilliance 221P3LPY display (resolution: 1920 X 1080, refresh rate: $60 \mathrm{~Hz}$ ). Visual stimuli were recorded with a Canon Legria HFS200 (30fps) and edited using MovieDek and Corel Paintshop Pro X6. Audio stimuli were recorded with a M-Audio Microtrack 2 Digital Voice Recorder and were delivered using a Logitech PC120 headphone set.

Stimuli. Eight videos of an arm reaching for an object were used, taken from our prior studies (Hudson et al., 2016 ${ }^{\mathrm{ab}}$ ). As in the previous experiments, half of the objects were safe to grasp, and half were painful to grasp to provide a meaningful context for the actor's statements of intending to grasp or withdraw. Firstly, four videos with safe objects were filmed from the actor's left, beginning with the actor's hand palm down on the table and reaching for an object placed in front of them. Each video was 1 second in duration and was separated into individual frames, producing 29 frames per action. For each of the sequences, the final 3 frames in which the actual grasp was performed were discarded, and each frame was numbered sequentially from 1 (the initial rest position) to 26 (just prior to contact with the object). All background details were replaced by a uniform black background. For each video, the safe object was digitally removed and replaced with an object that was of comparable size and shape but which was painful to grasp. This created a further four action sequences that were matched for reach trajectory and object size within each category of object type. Importantly, the verbal expression of intent on which our analysis was focused was fully balanced across actions, so subtle differences in movement kinematics cannot explain our results.

All action sequences were derived from this set. Each sequence began with a still frame taken from the middle portion of the sequence (randomly chosen between frames 11 and 16 on each trial), as if the actor was poised to either reach for or withdraw from the object. Importantly, as the start position was the same for each action direction, participants could not predict the direction of the action from the position in which it started. The action sequence depicted either 3, 4 or 5 frames 
either progressing forward through the frame sequence for reaches (e.g. 13-15-17) or backwards through the frame sequence for withdrawals (e.g. 13-11-9), each being present for 80ms, before it was replaced with the next frame (total duration of $240 \mathrm{~ms}, 320 \mathrm{~ms}$, or $400 \mathrm{~ms}$ ). The stimulus occupied a visual angle of $.07 \mathrm{X} .12$ degrees. The vertical position of the stimulus was aligned with the horizontal midline. The horizontal position varied across trials between $250 \mathrm{px}$ to the left or right of the vertical midline.

The probe stimulus was a single frame depicting the hand and the object taken from the same frame sequence as the action. The frame could either be the same as the final frame of the action sequence ( 0 frame difference), or shifted by 1 or 2 frames either up or down the frame sequence, thus depicting the action as nearer to or farther from the object. Therefore, in relation to the direction of motion in the action sequence, the probe would depict a position that would've been predicted to occur had the action continued (nearer the object for reaches, farther from the object for withdrawals, denoted by “+”), or depict an unpredicted change in direction (farther from the object for reaches, nearer the object for withdrawals, denoted by ““_"). This resulted in five possible probe displacements with respect to the final frame of the action ( -2 frames, -1 frame, 0 frame, +1 frame, +2 frames).

Audio stimuli. Two recordings of an actor saying the statements "I'll take it" and "I'll leave it", each of $1000 \mathrm{~ms}$ duration. The audio stimuli were presented to participants with a $50 \%$ bias to the right ear to match the actor's position on the right of the screen.

Procedure. Each trial (Figure 1) began with a fixation cross (duration randomly chosen between 500 and 1000ms on each trial) followed by a blank screen (duration 1000ms minus fixation cross duration). The first frame of the action stimulus was then shown and, after a randomly chosen interval of between 1000 and 3000ms, the intention statement ("I'll take it" or "I'll leave it") was heard. Two-hundred ms after the end of the intention statement, the action sequence began. The short and constant interval between audio stimulus and action onset created the impression of 
causality between the intention and the ensuring behavior, especially in contrast to the long and variable interval before onset of the audio stimulus. A blank screen was then presented for $250 \mathrm{~ms}$ (the optimal interval by which to observe a predictive perceptual bias, Freyd \& Johnson, 1987) to give the impression that the arm and object had suddenly disappeared. The probe stimulus was then presented which depicted the hand and object in either the same position as the final frame of the action stimulus ( 0 frame difference), shifted further forward along the observed trajectory by 1 frame (+1) or 2 frames (+2), or shifted backward in the opposite direction of motion by 1 frame $(-1)$ or 2 frames (-2). Participants pressed the spacebar if they thought the probe was in a different position to that of the disappearance point of the action, or refrained from responding if they thought the positions were the same. The probe stimulus was presented for a maximum of $4000 \mathrm{~ms}$ or until a response was made.

Design. Participants completed two blocks of 80 trials consisting of iterating the factors Object Type (2) X Intention (2) X Probe (5) X 4 repetitions (randomly presented within each block). The specific object was randomly chosen on each trial. For the Predictive group, the action was congruent with the intention for three repetitions and was incongruent for one repetition (120 congruent trials vs. 40 incongruent trials across the experiment). Participants were told that after hearing the intention, the actor will most likely do as they had said (if they said "I'll take it", they would most likely reach for the object, if they said "I'll leave it" they will most likely withdraw from it). For the Counter-predictive group, the action was incongruent with the intention for three repetitions and congruent for one repetition (120 incongruent trials vs. 40 congruent trials across the experiment). Participants were told that after hearing the intention, the actor will most likely do the opposite of what they had said (if they said "I'll take it", they would most likely withdraw from the object, if they said "I'll leave it" they will most likely reach for it).

The experiment contained eight catch trials per block in which the probe stimulus was displaced by $+/-4$ frames from the disappearance point. These trials were sufficiently obvious that 
the difference could be identified with ease, and were used to screen participants who did not comply with or understand the task instructions. Participants first completed six practice trials. The probe was displaced by $+/-3$ frames from the disappearance point of the action stimulus, such that the task could be learnt with ease. For the practice and catch trials, the intention and action were always congruent for the Predictive group, and incongruent for the Counter-predictive group.

Participant Exclusions. Participants were excluded from analysis if their accuracy in the catch trials was less than 3SD of the mean accuracy, suggesting they were insensitive to even the largest probe differences. Participants were also excluded if their catch trial accuracy was no better than their ability to detect the different probes in the experimental trials (catch trial accuracy minus experimental trial accuracy $<0$ ), suggesting an insensitivity to the varying task difficulties caused by the probe stimuli, and an essentially flat response profile.

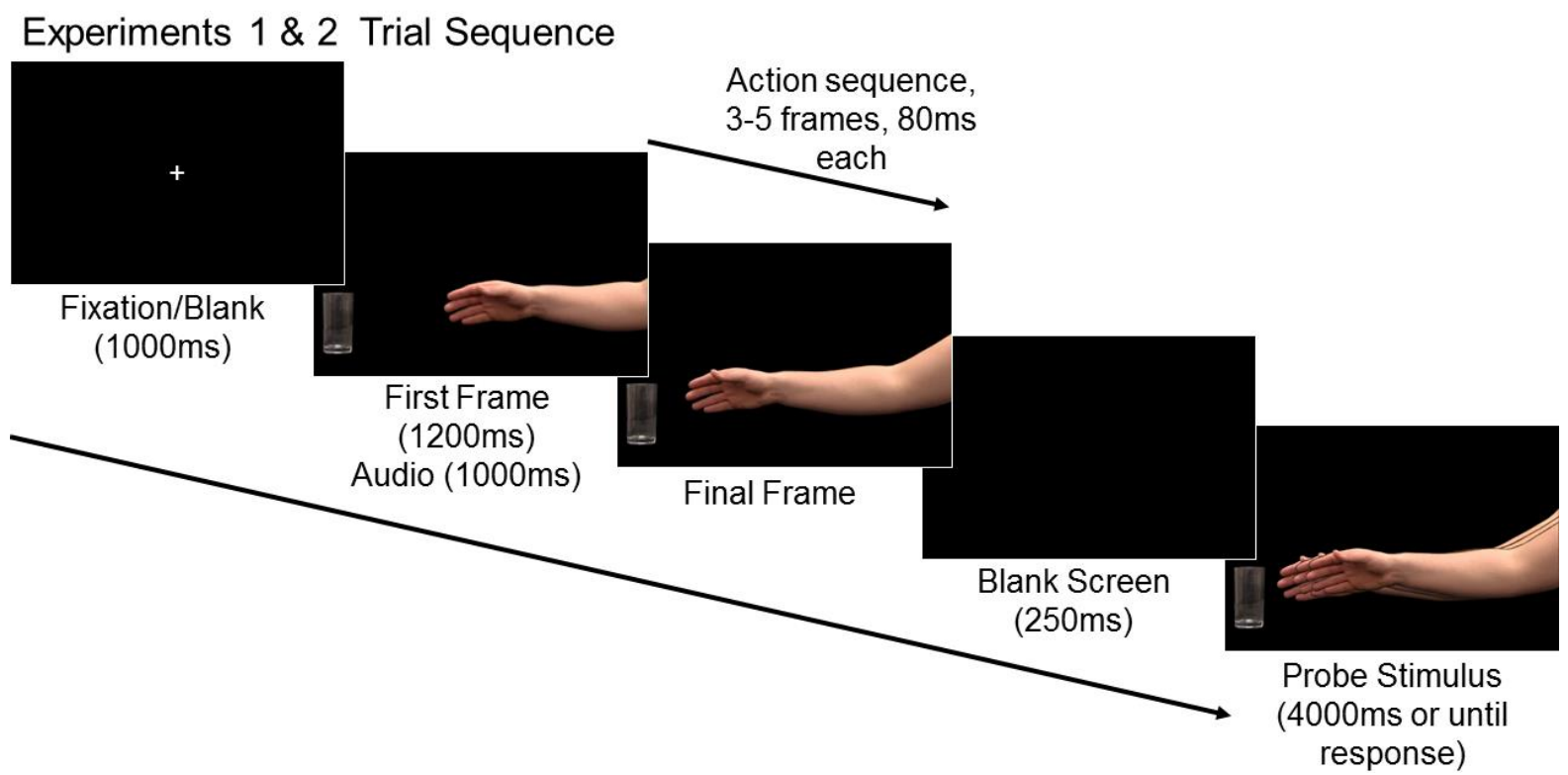

Figure 1. Trial sequence for Experiments 1 and 2. After a fixation cross and blank screen, participants observed a still image of a hand and an object and heard the actor state an intention ("I'll take it" or "I'll leave it"). The action sequence then followed depicting the actor either reaching for the object (shown) or withdrawing from the object. After a brief blank screen, participants observed a static probe stimulus depicting the actor in one of three positions (superimposed here for illustrative purposes): (i) the same as the final frame (center), (ii) further along the predicted path of motion (nearer the object for reaches, farther from the object for withdrawals), or (iii) in the opposite direction in an unpredicted position (further away from the object for reaches, nearer the object for withdrawals). The distance of the probes from the real final position in this figure is 2 frames. 


\section{Results}

The mean catch trial accuracy was $88 \%(S D=12)$ and the mean sensitivity was $37 \%(S D=$ 18 ), and the two groups did not differ in either measure $(t(76),=.244, p=.808$ and $t(76)=1.06, p=$ .292 respectively). Three participants were excluded from the Predictive group. As in our prior studies (Hudson et al., 2016 ${ }^{\mathrm{ab}}$ ), analysis was conducted on the predictive perceptual bias itself, by subtracting the mean percentage of different responses for the forward probes from that of the backward probes for each condition. A positive value denotes that observers were more likely to perceive backward probes as being different from the hand's last seen position than forward probes, and therefore provides an index of the predictive perceptual bias. The predictive biases in each condition were entered into a mixed measures ANOVA with Group (Predictive vs. Counterpredictive) as a between subjects factor, and Intention ("I'll take it" vs. "I'll leave it"), Action (reach vs. withdraw), and Block (first vs. second) as within subjects factors (the latter tested for the possible emergence or decay of any effects $)^{1}$.

The influence of Intention and Action on the size of the predictive perceptual bias (percentage of different responses for backward probes - forward probes) for both groups is shown in Figure 2. As can be seen, the perceptual biases were positive in all conditions, showing the expected perceptual displacement in the direction of motion. One sample t-tests revealed that each was larger than zero (all $\mathrm{p}<.0125$ ), with 95\% confidence intervals not overlapping with zero. Importantly, the ANOVA carried out on these predictive biases revealed a two-way interaction of Intention and Action $\left(F(1,73)=22.8, p<.001, \eta_{p}{ }^{2}=.238\right)$. The predictive perceptual bias was larger for actions that were congruent with the intention than actions that were incongruent, replicating our prior studies (Hudson et al., 2016 ${ }^{\mathrm{ab}}$ ). Importantly, as predicted, this two-way interaction was further modulated by $\operatorname{Group}\left(F(1,73)=8.92, p=.004, \eta_{p}^{2}=.109\right)$. The intention statements affected the predictive perceptual bias more strongly in the Predictive than the Counter-

\footnotetext{
${ }^{1}$ At a reviewer's request, and to simplify the presentation of the results, the factor of Object Type was excluded from analysis as it did not reveal any theoretically relevant effects, which is consistent with previous studies (Hudson et al, $\left.2016^{\mathrm{ab}}\right)$.
} 
predictive group. Indeed, step-down analyses revealed an effect of Intention-Action congruency on the predictive perceptual bias in the Predictive group $\left(F(1,36)=34.9, p<.001, \eta_{p}{ }^{2}=.492\right)$ but not the Counter-predictive group $\left(F(1,37)=1.52, p=.226, \eta_{p}^{2}=.039\right)$. In the Predictive group, the predictive perceptual bias for reaches toward the object were larger after hearing "I'll take it" than "I'll leave it" $(t(36)=4.82, p<.001, d=.62,95 \%$ CI $[10,24])$, whilst the predictive perceptual bias for withdrawals was larger after hearing "I'll leave it" than "I'll take it" $(t(36)=2.86, p=.007, d=$ $.38,95 \%$ CI $[3,18])$. Finally, Block did not modulate the effects (Block*Intention*Action: $F(1,73)$ $=.565, p=.455, \eta_{p}{ }^{2}=.008$; Block*Intention*Action*Group: $\left.F(1,73)=.872, p=.353, \eta_{p}{ }^{2}=.012\right)$. There were no further main effects or interactions (all $F<2.17$, all $p>.145$ ).

\section{Experiment 1: The effect of stimuli \& instructions}
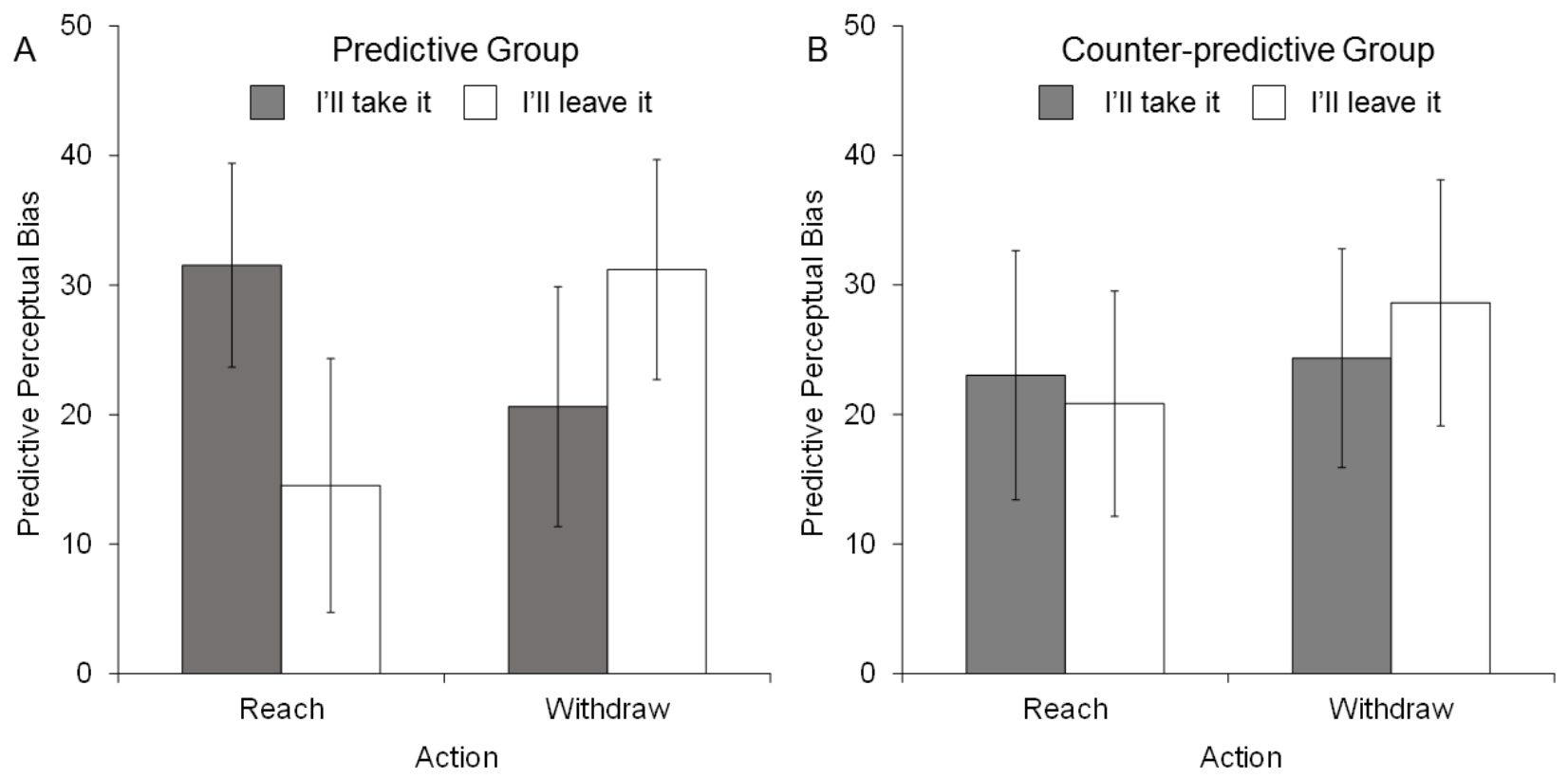

Figure 2. The intention-action prediction effect when the Intention is either predictive (A) or counter-predictive (B) of the action. Each bar depicts the predictive perceptual bias (different responses for backward probes minus forward probes), and therefore how much more likely a probe displaced forward in time is perceived as the same as the current position than one displaced an equal distance backward in time. The predictive perceptual bias is depicted for each action (reach, withdraw) after hearing each intention (I'll take it, I'll leave it). The actions were either congruent with the prior intention (outer bars) or incongruent (inner bars). Error bars represent $95 \%$ confidence intervals. 


\section{Experiment 1 Discussion}

The results confirmed our predictions. Firstly, they replicated the classic predictive perceptual bias in motion perception (representational momentum: Freyd \& Finke, 1984; Hubbard, 2005). In all conditions, the reported action disappearance points were shifted farther along the observed trajectory than they really were. An action reaching for the object was perceived as nearer the object, whereas an action withdrawing from the object was perceived as farther away. Furthermore, fully replicating our prior work (Hudson et al., 2016 ${ }^{\mathrm{ab}}$ ), this predictive bias was larger for actions that were correctly predicted by the actor's intention than for actions that contradicted the intention, such that hands reaching for objects were judged to have travelled further when the actor had just stated a goal to reach ("I'll take it") compared to a goal to withdraw ("I'll leave it"), and vice versa for withdrawals from the same objects. This finding confirms that motion prediction is not only driven by low-level motion cues, but is directly influenced by prior knowledge of the actor's intentions. Finally, and most importantly, this intention-action prediction effect was itself modulated by whether the intention was predictive of the actor's action: it was greater when the intention was predictive of the forthcoming action than when the intention was counter-predictive. Varying the predictability of an actor's action from their intention therefore elicits a corresponding variation in the predictive perceptual bias, such that highly reliable cues have larger effects on the action's perceptual representation compared to cues for which these predictive relationships are undermined.

\section{Experiment 2}

Revisions of internal predictive models can be driven either by explicit processes, such as the declarative knowledge we have - or are given - about others (e.g., gossip, Beersma \& van Kleef, 2012), or by more implicitly learned information, from the statistical relationships between cues that others give away and the actions they subsequently perform. The following experiments 
( $2 \mathrm{a}$ and $2 \mathrm{~b}$ ) disentangled the contributions of these two processes, and established whether the intention modulation of the perceptual judgments was due to explicit information about these contingencies or due to the actual experience of observing them.

Experiment $2 \mathrm{a}$ tested the role of statistical relationships in isolation. It used the same statistical relationships between intention cues and actions as Experiment 1 but participants were not instructed about the differing intention-action contingencies. Despite one group experiencing a $75 \%$ congruency rate (Predictive Contingencies group), and a second group experiencing a 25\% congruency rate (Counter-predictive Contingencies group), both groups were instructed that there was no relationship between the intention they would hear and the following action. The instructions therefore followed those of Hudson et al (2016 $)$, who reported an intention-action prediction effect when the intention was non-predictive of the action (50\% congruency rate).

In contrast, Experiment $2 \mathrm{~b}$ tested the role of explicit information in isolation. It presented both groups with a 50\% congruency rate between intention and action, such that actors were just as likely to reach for an object than to withdraw after hearing "I'll take it" or "I'll leave it" (as per Hudson et al, 2016 ${ }^{\mathrm{a}}$. However, the instructions given to each group of participants matched those of Experiment 1. One group was told that the intention was predictive and that the actor would most likely do as they had said (Predictive Instructions group), whilst the other group was told that the intention was counter-predictive and that the actor would most likely do the opposite of what they had said (Counter-predictive Instructions group).

There are three potential outcomes. If the intention-action prediction effect in Experiment 1 required there to be an actually predictive relationship between the intention and action, then the results will be replicated in Experiment 2a. If the results of Experiment 1 are driven by top-down knowledge about the contingencies, then Experiment $2 b$ will replicate the group differences. Finally, it is possible that internal models are only updated if explicit hypotheses about intentionaction contingencies can be confirmed by the statistics of the perceptual input (e.g., Dale, Duran \& 
Morehead, 2012; Duran \& Dale, 2009; Marcus, Karatekin, \& Markiewicz, 2006). This might be particularly likely if one assumes that adjusting the precision of a prediction is relatively resource intensive and requires tracking and integrating prediction errors across multiple events (Hohwy, 2013). In situations where predictions are task irrelevant such as here (see also Schenke, Wyer \& Bach, 2017; Joyce et al., 2014), they might therefore not be undertaken if not prompted by explicitly given information. No group differences in intention modulation of the predictive perceptual bias would then be expected in either, where such a re-evaluation would either not be triggered (Experiment 2a) or fail due to conflicting perceptual input (Experiment 2b).

\section{Experiment 2a: The effect of intention-action contingencies only}

\section{Method}

Participants. A power analysis revealed that a sample of at least 32 per group is required to achieve the same effect size for the between-group difference in the intention-action prediction effect of Experiment 1 with a power of 0.8. We tested more than this to account for potential exclusions. Seventy two participants took part in Experiment 2a (Predictive Contingencies group $n$ $=36$, Counter-predictive Contingencies group $n=36$, mean age $=21.2$ years, $S D=4.8,60$ females). The groups did not differ in terms of age $(t(69)=1.31, p=.196)$ or gender ratio $\left(X^{2}{ }_{1}=\right.$ $.077, p=.782$ ) (gender and age information was not recorded for one participant). All other participant details are the same as for the previous experiment.

Procedure. All stimuli, apparatus and procedural aspects were the same as in Experiment 1. Only the verbal and written instructions given prior to the experiment differed. Both groups were told that, after hearing the actor state an intention of "I'll take it" or "I'll leave it", the actor was just as likely to do as they said as do the opposite of what they said. 


\section{Results}

Data were cleaned and analyzed as in Experiment 1. The mean catch trial accuracy was $88 \%$ $(S D=14)$ and the mean sensitivity was $35 \%(S D=19)$. The groups did not differ in terms of sensitivity $(t(70)=1.21, p=.232)$ but the Predictive Contingencies group were marginally $(t(70)=$ $1.76, p=.082)$ more accurate in the catch trials $($ mean $=90 \%, S D=12)$ than the Counter-predictive Contingencies group (mean $=85 \%, S D=15)$. One participant from the Predictive Contingencies group and four participants from the Counter-predictive Contingencies group were excluded.

The results (Figure 3a \& b) confirmed, as in Experiment 1, the classic representational momentum effect, with positive perceptual biases in all conditions in both groups. Indeed, one sample t-tests showed that the predictive perceptual biases were larger than zero in all conditions (all $\mathrm{p}<.0125$ ), and confidence intervals did not include zero. As before, the predictive perceptual biases were analyzed with a mixed measures ANOVA with Group (Predictive vs. Counterpredictive) as a between subjects factor, and Intention ("I'll take it" vs. "I'll leave it"), Action (reach vs. withdraw), and Block (first vs. second) as within subjects factors. Replicating Experiment 1 and Hudson et al., 2016 ${ }^{\mathrm{ab}}$, this analysis revealed a two-way interaction between Intention and Action $\left(F(1,65)=15.2, p<.001, \eta_{p}{ }^{2}=.190\right)$, showing a larger predictive perceptual bias for actions that correspond to the stated intention. However, in contrast to Experiment 1, there was no evidence that this effect was further modulated by $\operatorname{Group}\left(F(1,65)=.017, p=.898, \eta_{p}{ }^{2}=.000\right)$. Indeed, the relevant two-way interaction between Intention and Action was present for both the Predictive Contingencies group $\left(F(1,34)=6.23, p=.018, \eta_{p}{ }^{2}=.155\right)$ and the Counter-predictive Contingencies group $\left(F(1,31)=9.56, p=.004, \eta_{p}{ }^{2}=.236\right)$, showing a larger predictive perceptual bias for actions congruent with the intention than for incongruent actions. Overall, the predictive perceptual bias for reaches was larger after hearing "I'll take it" than "I'll leave it" $(t(66)=3.17, p=$ $.002, d=.35,95 \%$ CI $[4,17])$, whilst the predictive perceptual bias for withdrawals was larger after hearing "I'll leave it" than "I'll take it" $(t(66)=2.92, p=.005, d=.29,95 \%$ CI $[3,14])$. 
Finally, there was a theoretically irrelevant interaction between Block and Group $(F(1,65)=$ $\left.7.16, p=.009, \eta_{p}^{2}=.099\right)$. As this effect was theoretically irrelevant and not predicted and therefore subject to alpha inflation it will not be interpreted further. There were no further main effects or interactions (all $F<2.36$, all $p>.130$ ).

\section{Experiment 2b: The effect of instructions only}

\section{Method}

Participants. Seventy three participants took part in Experiment 2b (Predictive Instructions group $n=34$, Counter-predictive Instructions group $n=39$, mean age $=22.4$ years, $S D=6.2,61$ females). The groups did not differ in terms of age $(t(71)=.071, p=.944)$ or gender composition $\left(X^{2}{ }_{1}=2.33, p=.127\right)$. All other participant details are the same as the previous experiments.

Procedure. All stimuli and apparatus were the same as in Experiments 1 and 2a. Both groups of participants were given the same experiment. Each participant completed two blocks of 80 trials (Object Type (2) X Intention (2) X Action (2) X Probe (5) X 2 repetitions). The Intention and Action were therefore 50\% congruent (non-predictive) for both groups. The congruency between Intention and Action in the training trials and catch trials was 50\%. All other procedural details were the same as Experiments 1. Notably, the Predictive Instructions group were given the same instructions as the Predictive group in Experiment 1 (the actor will most likely do as they said) whereas the Counter-predictive Instructions group were given the same instructions as the Counter-predictive group of Experiment 1 (the actor will most likely do the opposite of what they said). 


\section{Results}

Data were cleaned and analyzed as before. The mean catch trial accuracy was $86 \%(S D=$ $16)$ and the mean sensitivity was $34 \%(S D=21)$, and the two groups did not differ in either measure $(t(71)=0.16, p=.987$ and $t(71)=.769, p=.444$ respectively). Three participants were excluded from the Predictive Instructions group and three from the Counter-predictive Instructions group.

As before, there were positive perceptual biases in all conditions in both groups, all of which were larger than zero (all $p<.0125$ ), and for which confidence intervals did not include zero (Figure $3 \mathrm{c} \& \mathrm{~d}$ ). As before, the ANOVA showed the predictive perceptual bias to be larger for actions that were congruent with the intention than those that were incongruent (Intention*Action: $\left.F(1,65)=9.14, p=.004, \eta_{p}{ }^{2}=.123\right)$. Withdrawals elicited a larger predictive perceptual bias if preceded by the intention of "I'll leave it" than "I'll take it" $(t(66)=2.52, p=.014, d=.21,95 \%$ $\mathrm{CI}[1,11])$, whereas reaches elicited a marginally larger predictive perceptual bias if preceded by "I'll take it" than "I'll leave it" $(t(66)=1.79, p=.079, d=.16,95 \%$ CI[0, 10]). However, as in Experiment 2a, there was no three-way interaction between Intention, Action, and Group $(F(1,65)=$ $\left..922, p=.341, \eta_{p}{ }^{2}=.014\right)$. The effect of intention-action congruency on the predictive perceptual bias was present numerically for the Predictive Instructions group $\left(F(1,30)=2.05, p=.163, \eta_{p}{ }^{2}=\right.$ $.064)$ and significant in the Counter-predictive Instructions group $\left(F(1,35)=8.29, p=.007, \eta_{p}{ }^{2}=\right.$ .192). There were no further main effects or interactions (all $F<3.53$, all $p>.065$ ). 
Experiment 2a: The effect of contingencies only
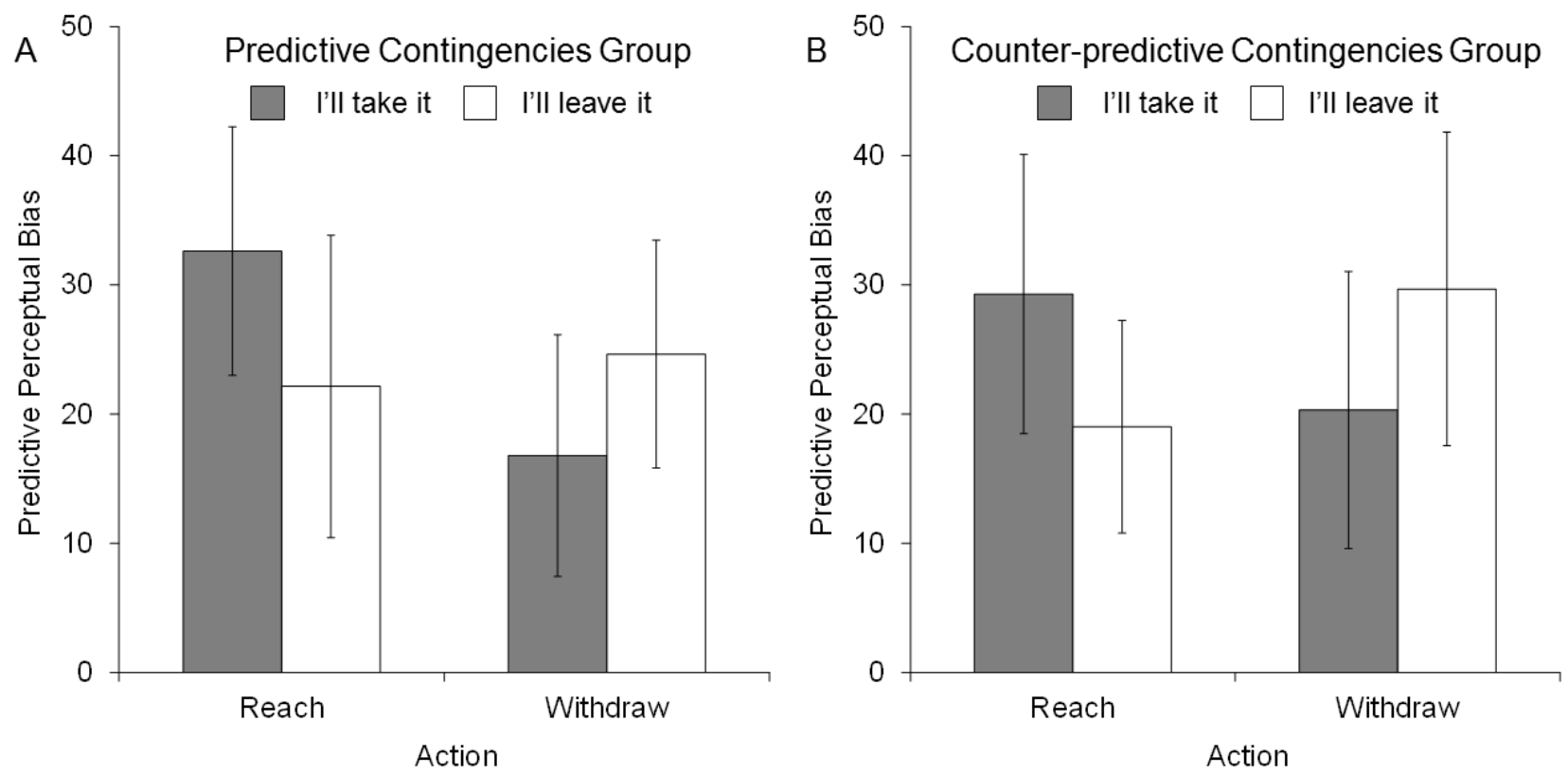

Experiment 2b: The effect of instructions only
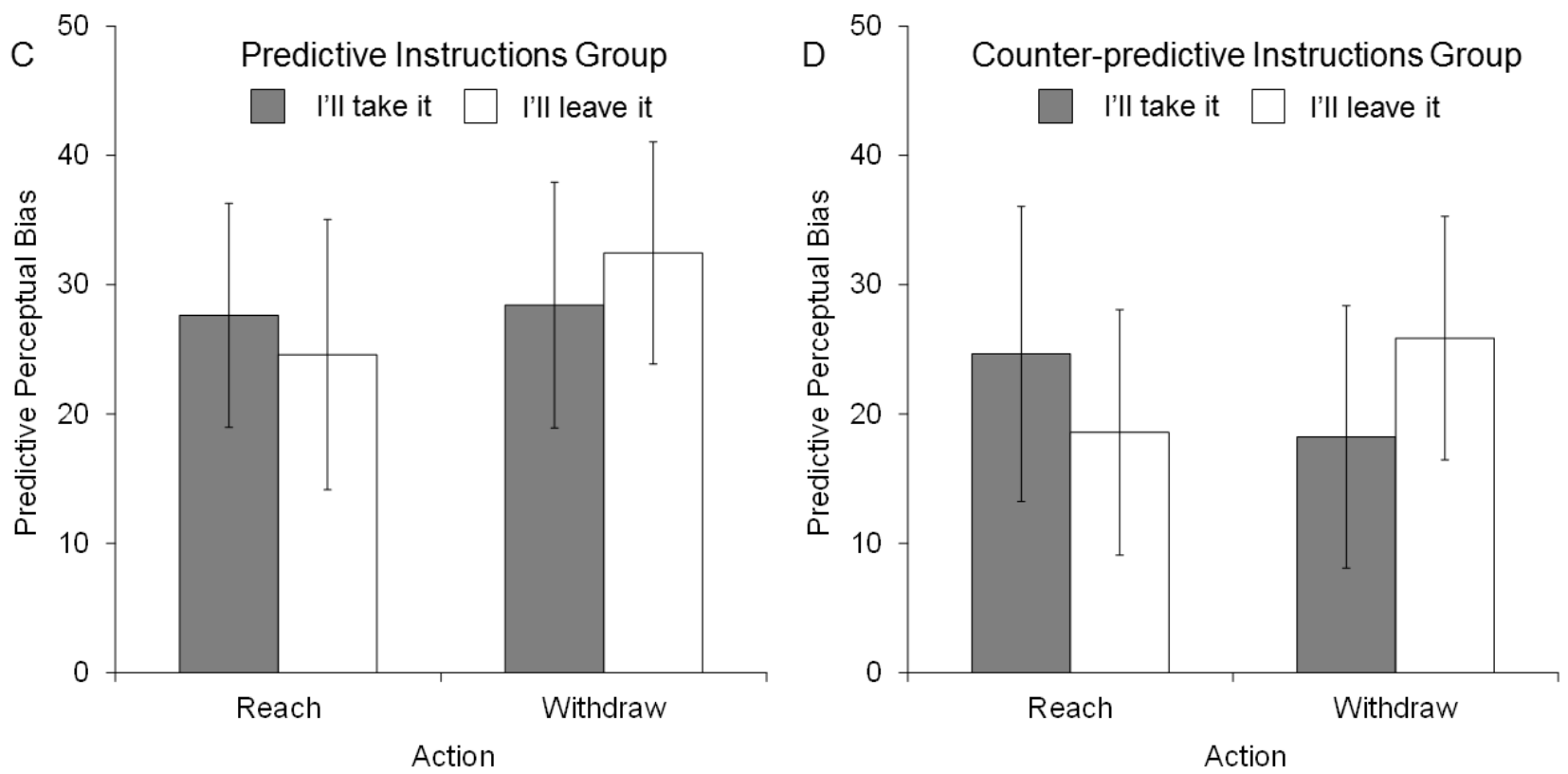

Figure 3. The Intention-action prediction effect for each group in Experiment 2. In Experiment 2a (top row) the intention was either predictive (A) or counter-predictive (B) of the action, but participants believed it to be non-predictive. In Experiment $2 b$ (bottom row), participants believed that a non-predictive relationship was in fact predictive (C) or counter-predictive (D). Error bars represent $95 \%$ confidence intervals. 


\section{Experiment 2 Discussion}

In all four groups, the predictive perceptual bias was larger for actions that were correctly predicted by the actor's intention than for actions that were incorrectly predicted, demonstrating again the robustness of the intention-action prediction effect, first demonstrated by Hudson and colleagues $\left(2016^{\mathrm{ab}}\right)$. However, neither group differed from one another, such that the prior intention statements had the same effect irrespective of whether participants were given explicit information about the reliability with which they predict the subsequent actions (while the actual relationships were equivocal), or when these relationships were present in the stimulus statistics (but not made explicitly salient). To further confirm this lack of influence, the data from Experiments $2 \mathrm{a}$ and $2 \mathrm{~b}$ were pooled to increase power to detect a difference in the intention-action prediction effect between the Predictive and Counter-predictive groups (power of .99 to detect equal effect size as Experiment 1) and a two-way ANOVA was conducted on the intention-action prediction effect (the Intention*Action interaction) with Predictability (Predictive vs. Counter-predictive) and Information (stimulus-contingencies vs. instructions) as between subjects factors. The intentionaction prediction effect did not interact with either Predictability $\left(F(1,130)=.476, p=.492, \eta_{p}{ }^{2}=\right.$ $.004)$, Information type $\left(F(1,130)=2.08, p=.151, \eta_{p}^{2}=.016\right)$, nor was there an interaction $\left(F(1,130)=.206, p=.651, \eta_{p}^{2}=.002\right)$.

Nevertheless, it could be argued that group differences were present in Experiments 2a and $2 \mathrm{~b}$, yet were undetectable due to a lack of power, making it difficult to draw a firm conclusion regarding the effect of instructions and/or stimulus statistics from these null effects. We therefore compared the group differences between the Predictive and Counter-predictive groups between Experiment 1 and the pooled data of Experiments $2 \mathrm{a}$ and $2 \mathrm{~b}$ by conducting a two-way between subjects ANOVA on the intention-action prediction effect with Experiment (1 vs 2) and Group (predictive vs counter-predictive) as factors. Indeed, there was a two-way interaction between Experiment and Group, $\left(F(1,205)=6.62, p=.011, \eta_{p}{ }^{2}=.031\right)$. This demonstrates that the 
difference between the predictable and counter-predictive groups on the intention-action prediction effect was larger in Experiment 1, than in Experiments 2a and 2b combined. This pattern also held when Experiment 1 was compared with Experiment $2 \mathrm{a}\left(F(1,138)=3.56, p=.061, \eta_{p}{ }^{2}=.025\right)$ and Experiment $2 \mathrm{~b}$ separately $\left(F(1,138)=7.85, p=.006, \eta_{p}{ }^{2}=.054\right)$. Therefore, even if a weak group difference were present in Experiments $2 \mathrm{a}$ and 2b, it was still smaller than in Experiment 1, demonstrating the stronger effect that combined knowledge and stimulus statistics have on predictive perception biases.

Together, these data show that simply having explicit information that the intention was predictive of the action was insufficient to shape the precision of the prediction, but neither did actual experience of these contingencies have an influence in the absence of this explicit knowledge. This can be seen in Figure 4, which depicts the intention-action prediction effect, or how much more predictive perceptual bias there was for congruent actions than incongruent actions (e.g. the size of the Intention*Action interaction) for each of the groups in Experiments 1 and 2, and how it was affected by predictive or counter-predictive relationships. Furthermore, there was no effect of experimental block, suggesting that any effect of explicit information did not affect perception early in the experiment and decline thereafter, nor did statistical exposure to the predictability emerge during the course of the experiment.

The data therefore indicate that for the precision of a prediction to be influenced by prior knowledge, the observer must be explicitly aware of the likelihood and this must be reinforced by experience. The strong influence of combined instruction and stimulus statistics is consistent with the assumption that such re-weighting of predictive relationships is resource-intensive and is, when these prediction have no bearing on task success such as here, are only undertaken when prompted by explicit information and when this information is reinforced by perceptual experience. It also dovetails nicely with recent findings from associative learning. While associative learning has previously been linked to highly automatic mechanisms, this assumption is increasingly in doubt 
(Shanks \& John, 1994), with recent studies showing that even classical implicit measures of contingency learning and conditioning rely on awareness and prior instruction (e.g., Pavlovian eyeblink conditioning) and are not obtained when participants are not guided towards the relevant stimulus dimensions (Weidemann, Satkunarajah, \& Lovibond, 2016; see Mitchell, De Houwer, \& Lovibond, 2009, for a review).

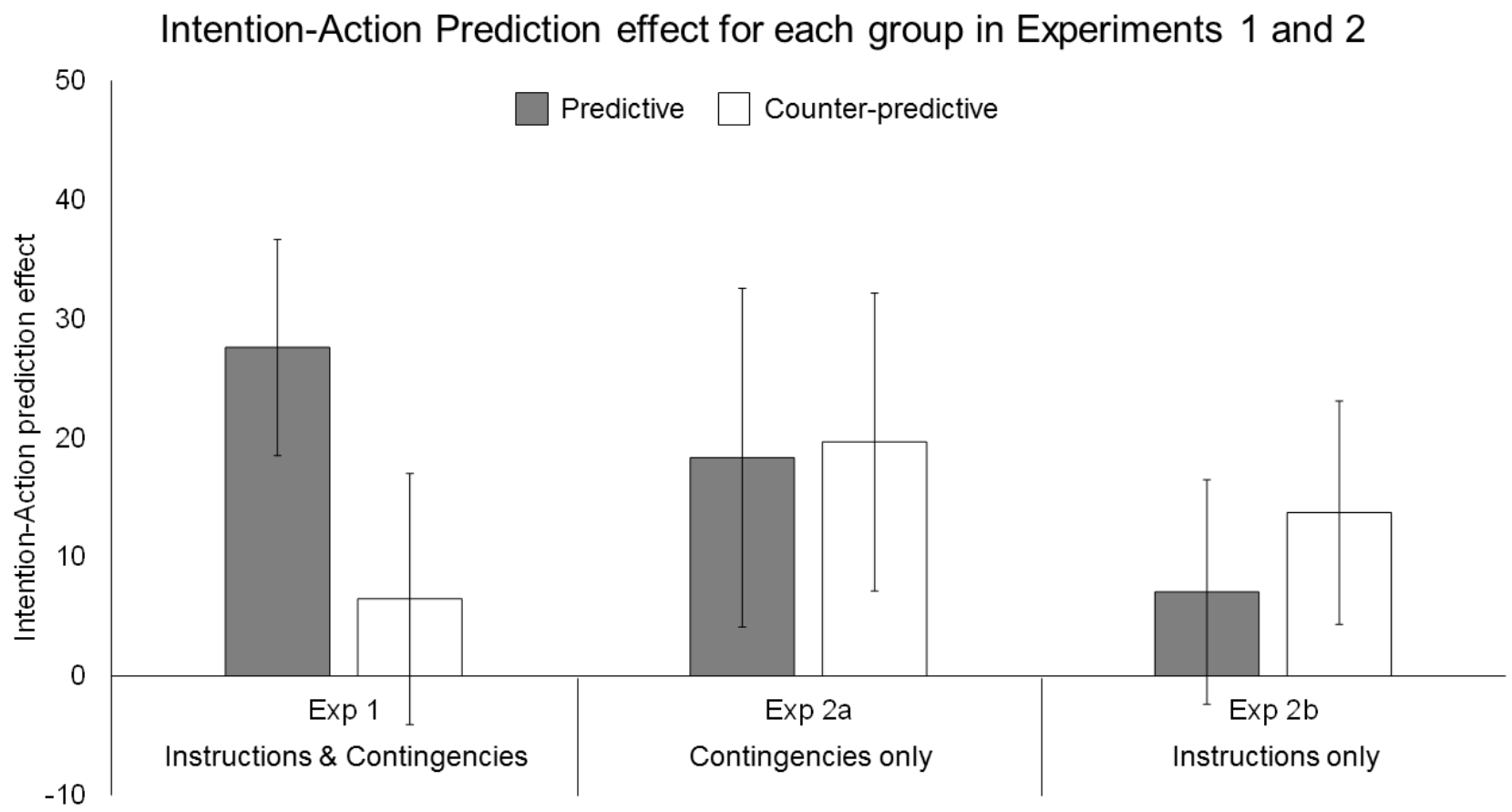

Figure 4. A comparison of the intention-action prediction effect for each group across Experiments 1 and 2. Each bar represents how much larger the predictive perceptual bias was for actions that were correctly predicted by the intention than actions that contradicted the intention. Error bars represent $95 \%$ confidence intervals.

\section{Experiment 3}

The previous results suggest that the intention-action relationship only modulates prediction if buttressed by explicit information, as in the case of Experiment 1 but not in Experiment $2 \mathrm{a}$ and $2 b$, where either the stimulus statistics or the explicit information undermined updating of prediction weights. The aim of Experiment 3 was to replicate and extend the results of Experiment 1 with an alternative method for measuring the predictive perceptual bias to ensure that its results were not due simply to random group differences unrelated to the experimental manipulations under 
investigation (type 1 error). Here, we measure participants' perceived disappearance point not through a probe detection task (as in the previous experiments), but on a touch screen, by asking participants to estimate the last seen position of the action by touching the screen location, which records the XY screen coordinate of the response. Typically, participants' touch screen judgments also overshoot the true disappearance point further along the stimulus's path of motion, revealing a similar predictive bias to that elicited with the use of probe stimuli in a typical representational momentum task (Pozzo, Papaxanthis, Petit, Schweighofer \& Stucchi, 2006; Saunier, Papaxanthis, Vargas \& Pozzo, 2008).

The use of a touch screen has several advantages. Firstly, it allows us to assess the robustness of the intention-action prediction effect across different techniques. Secondly, the touch screen method affords increased power by obtaining a measure of predictive bias on each and every trial, unlike with the use of probes in which the predictive perceptual bias is indexed by comparing forward and backward probes across different trials, whilst ignoring the same probes. Thirdly, touch screens allow for a direct measure of the size of the perceptual overshoot in pixels, providing a more sensitive measure of the size of the predictive bias. Finally, the use of touch screens allows us to estimate perceptual displacements along both the $\mathrm{X}$ and $\mathrm{Y}$ axis, testing whether the reported forward biases reflect predictions of the expected reach trajectories (i.e. forward and up in case of a reach, and backwards and down in case of a withdrawal) or more basic left and rightward biases towards and away from objects.

The increased power and sensitivity allowed us to test whether the effect of predictability on action perception could be manipulated within participants. That is, if participants exhibit a large intention-action prediction effect in response to a highly predictive relationship, will this reduce if the relationship is suddenly changed to become counter-predictive? This would provide evidence that the precision of our predictions are under constant revision and can be done so immediately in light of new experience and knowledge. To this end, participants completed two blocks of trials, 
each presenting either a predictive or counter-predictive relationship between intention and action, and participants were explicitly informed about this relationship.

\section{Method}

Participants. Thirty-seven participants took part in the experiment (33 females, mean age $=$ 20.9 years, $S D=6.7)$. All other participant details are the same as previous experiments.

Stimuli and Apparatus. All stimuli were the same as the previous experiments, except that instead of the probe stimuli, a series of response stimuli were created. For each action sequence, a single frame was taken and the hand was erased to depict just the object, on which participants indicated where they perceived the action to have disappeared. The same apparatus were used except that a Hewlett Packard s230tm EliteDisplay Touchscreen (1920 x 1080 resolution, $60 \mathrm{~Hz}$ refresh rate) was used to present visual stimuli and record touch screen responses.

Procedure. Each trial (Figure 5) began with a written instruction of "Hold the spacebar", to which participants pressed the spacebar down with their right hand and kept it pressed until a response was required. This was to ensure that participants did not track the motion of the action with their finger. Once they had done so, a fixation cross was presented $(500-1000 \mathrm{~ms})$, and then the first frame of the action sequence (between frames 11 and 16). After a randomly chosen delay of between $1000-3000 \mathrm{~ms}$, the auditory stimulus was presented (1000ms), and the action sequence began $200 \mathrm{~ms}$ after auditory offset, and proceeded as per the previous experiments. The response stimulus was then presented, creating the impression that the action had simply disappeared leaving just the object. As soon as the action disappeared, participants released the spacebar and, with the same hand, touched the screen location of the last position of the tip of the index finger.

Design. Participants completed two blocks of 192 trials, each iterating the factors of object type (safe X 4, painful X 4), Intention (“I'll take it", "I'll leave it"), and action length $(3,4,5)$ X 4 repetitions. In the predictive block, the action was congruent with the intention for three repetitions 
(144 trials) and incongruent for one repetition (48 trials). In the counter-predictive block the action was incongruent with the intention for three repetitions (144 trials) and congruent for one repetition (48 trials). The order of trial presentation within each block was randomized and the presentation of the blocks were counter-balanced across participants. Before the predictive block, participants were told that the actor will most likely do as they had said, and completed eight practice trials in which the action was always congruent with the intention. Before the counter-predictive block participants were told that the actor will most likely do the opposite of what they had said, and completed eight practice trials in which the action was always incongruent with the intention.

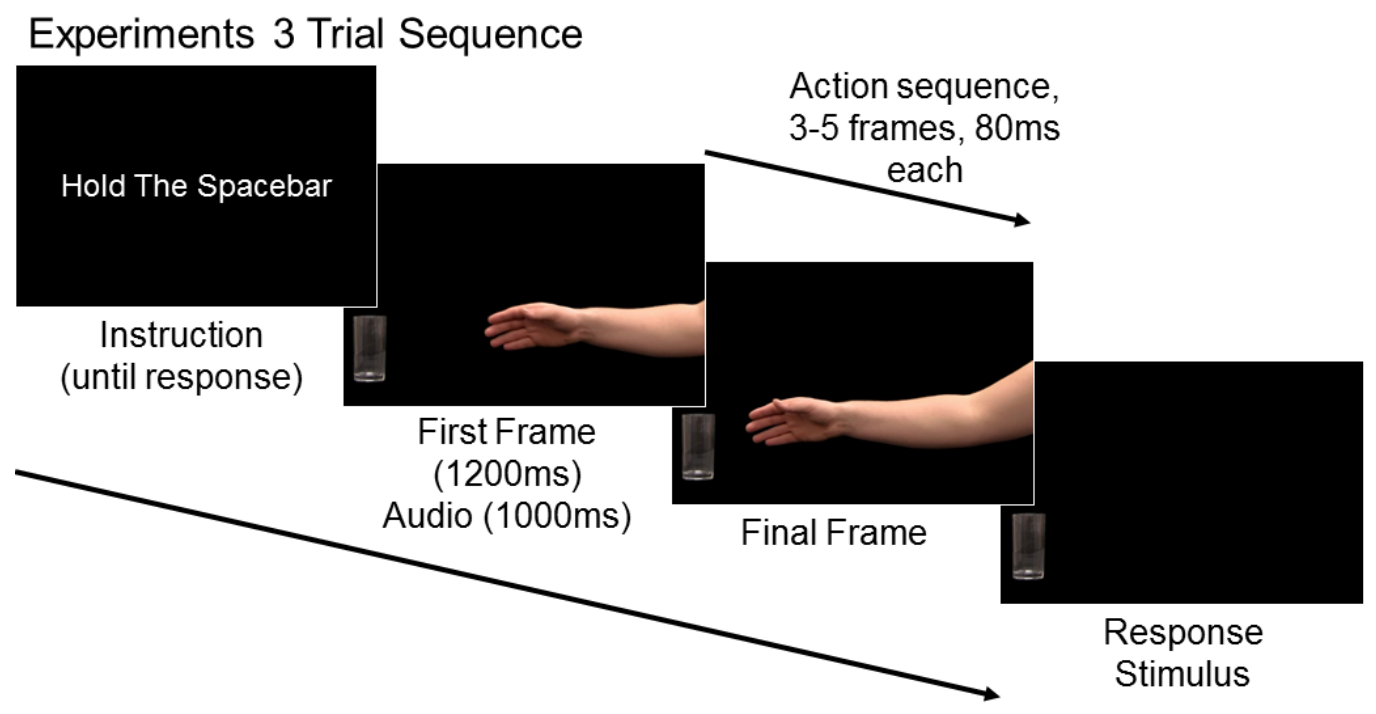

Figure 5. Trial sequence in Experiment 3. Participants were instructed to hold the spacebar, after which they saw the first frame of the action sequence and heard the actor's intentions. The action sequence of a reach (shown) or withdrawal was then presented, followed by an image of just the object (as if the hand had disappeared) upon which participants lifted the spacebar and tapped where the final position of the tip of the index finger had been.

\section{Results}

Participant exclusions. One participant was excluded from the dataset prior to analysis due to an inability to comprehend the task. Further participants were excluded if their responses bore little relationship to the real final position, as assessed by three a priori defined indices. Firstly, participants were excluded if the mean distance of their responses from the real position exceeded 
3SD above the group mean $($ mean $=119.3 \mathrm{px}, S D=24.8)$. No participants were excluded on this basis. Secondly, they were excluded if their responses did not significantly correlate with the real position on either the $\mathrm{X}$ axis or the $\mathrm{Y}$ axis. All participants exhibited a significant correlation between real and selected positions (X axis: median $r^{2}=.975, S D=.022 ; \mathrm{Y}$ axis: median $r^{2}=.859$, $S D=.087)$. Thirdly, two participants were excluded as the correlation between their selected position and the real position exceeded 3SD below the group median $\mathrm{r}^{2}$ on either the $\mathrm{X}$ axis or the $\mathrm{Y}$ axis. Individual trials were excluded (mean $=14.5 \%, S D=13.8)$ if participants did not follow the correct response procedure of holding the spacebar until a response was required, or if their response initiation time $($ mean $=376.2 \mathrm{~ms}, S D=93.0)$ or execution time $($ mean $=798.2 \mathrm{~ms}, S D=$ 168.7) was less than $200 \mathrm{~ms}$ or more than $3 \mathrm{SD}$ above the group mean.

Data analysis. The real final position of the tip of the observed hand's index finger was subtracted from the selected final position on the $\mathrm{X}$ axis and $\mathrm{Y}$ axis separately to leave the residual error in judging the final position of the action. A value of 0 on both axes represents that participants detected the real final position on any given trial. On the $\mathrm{X}$ axis, positive values denote a bias to the right, and negative values a bias to the left. On the $\mathrm{Y}$ axis, positive values denote a bias upwards, and negative values denote a bias downwards. Overall, there were significant response biases rightward $($ mean $=28.4 \mathrm{px}, S D=17.4, t(33)=9.41, p<.001, d=2.26, \mathrm{CI} 95 \%[22.5,34.3])$ and downward $($ mean $=-110.2 \mathrm{px}, S D=25.4, t(33)=25.3, p<.001, d=6.2, \mathrm{CI} 95 \%[-101.7$, 118.8]), towards the hand's center of gravity, as expected from prior research (Coren \& Hoenig, 1972).

The response biases in each condition were entered into a within subjects ANOVA for the $\mathrm{X}$ axis and $\mathrm{Y}$ axis separately with Intention-Action predictability (predictive, counter-predictive), Intention ('I'll take it", "I'll leave it") and Action (reach, withdrawal) as factors (Figure 6). A replication of the intention-action prediction effect requires that on the $\mathrm{X}$ axis there is a main effect of Action, reflecting the overall predictive perceptual bias (disappearance points of reaches shifted 
leftward, withdrawals shifted rightward), and a main effect of Intention (leftward shift after "I'll take it", rightward shift after "I'll leave it"). These two main effects result in a maximal shift left (nearer the object) for reaches after hearing "I'll take it" and a maximal shift right (farther from the object) for withdrawals after hearing "I'll leave it". This pattern of results would replicate those of Experiments 1, in which a larger positive predictive perceptual bias is observed for actions congruent with the intention. Unlike the previous experiment, no interaction is required to demonstrate an intention-action prediction effect because the perceptual bias recorded with touch screens is directionally specific (e.g. left, right). In contrast, the use of probes elicits a positive predictive perceptual bias that is always in the direction of motion, irrespective of which direction that is. An interaction is therefore required to demonstrate that this positive value is larger when the action is congruent with the intention than incongruent, for both actions.

$\mathbf{X}$ axis. There was a main effect of Action $\left(F(1,33)=16.7, p<.001, \eta_{p}{ }^{2}=.336,95 \%\right.$ CI $[7.4$, 21.1]), showing the expected predictive perceptual bias (i.e. representational momentum). The disappearance point of reaches were perceived farther left than withdrawals. Consistent with the previous experiments, there was a main effect of Intention $\left(F(1,33)=35.3, p<.001, \eta_{p}{ }^{2}=.517\right.$, $95 \%$ CI $[2.9,5.8])$ with the perceived disappearance point displaced farther left (towards the object) if the actor had said "I'll take it" than "I'll leave it". Importantly, as in Experiment 1, the effect of Intention interacted with Intention-Action predictability $\left(F(1,33)=10.6, p=.003, \eta_{p}{ }^{2}=.243,95 \%\right.$ CI $[1.7,6.8])$. The effect of Intention was larger when the intention was predictive of the action $\left(F(1,33)=38.9, p<.001, \eta_{p}^{2}=.541,95 \% \mathrm{CI}[4.5,8.5]\right)$ than when the intention was counterpredictive of the action $\left(F(1,33)=6.2, p=.018, \eta_{p}{ }^{2}=.159,95 \%\right.$ CI $\left.[0.5,4.1]\right)$.

None of the other, theoretically irrelevant, effects were significant. The effect of Action did not interact with Intention-Action predictability $\left(F(1,33)=1.51, p=.227, \eta_{p}{ }^{2}=.044,95 \%\right.$ CI $[-1.7$, 10.2]). The effect was equally large for both the predictive $\left(F(1,33)=9.56, p=.004, \eta_{p}{ }^{2}=.225\right.$, $95 \% \mathrm{CI}[4.5,20.2])$ and counter-predictive blocks $\left(F(1,33)=20.2, p<.001, \eta_{p}{ }^{2}=.379,95 \% \mathrm{CI}\right.$ 
$[9.1,23.1])$. There was no interaction between Action and Intention $\left(F(1,33)=.01, p=.921, \eta_{p}{ }^{2}=\right.$ $.000,95 \%$ CI $[-3.0,3.4])$.

Y axis. There was a main effect of Action $\left(F(1,33)=205, p<.001, \eta_{p}{ }^{2}=.861,95 \% \mathrm{CI}\right.$ $[36.7,48.4])$ with the perceived disappearance point higher for reaches than for withdrawals, showing again the expected predictive perceptual bias as reaches were biased upwards. The effect of Action interacted with Intention-Action predictability $\left(F(1,33)=8.77, p=.006, \eta_{p}{ }^{2}=.210,95 \%\right.$ CI $[2.0,9.7])$. The effect of Action was larger when the intention was predictive of the action $\left(F(1,33)=214.3, p<.001, \eta_{p}^{2}=.867,95 \%\right.$ CI $\left.[39.4,51.6]\right)$ than when counter-predictive $(F(1,33)$ $=157.5, p<.001, \eta_{p}{ }^{2}=.827,95 \%$ CI $\left.[33.4,45.8]\right)$. There were no further main effects or interactions (all $F<1.51$, all $p>.227$ ).

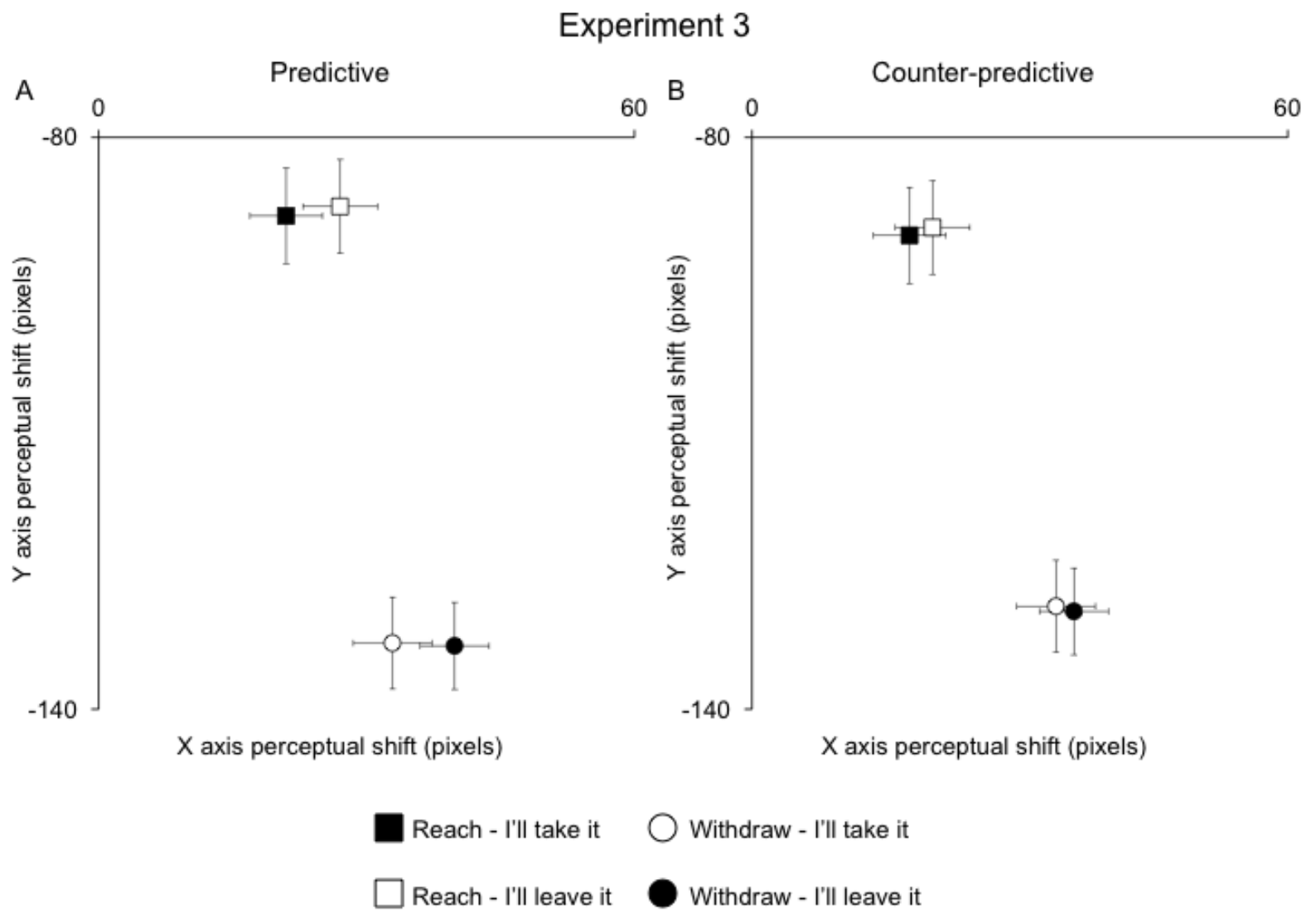

Figure 6. The perceptual shift elicited by the intention-action prediction effect when the intention was predictive (A) or counter-predictive (B) of the action. Values represent the difference between the selected disappearance point and the real disappearance point in pixels. Error bars represent standard error of the mean. 


\section{Experiment 3 Discussion}

Experiment 3 fully replicated and extended the results of Experiment 1. Firstly, overall, the perceived disappearance points of the hand were displaced further along the observed trajectory, along both the $\mathrm{X}$ and $\mathrm{Y}$ axis. Reaches were reported as above and left of the real position, and withdrawals were reported as below and right of the real position (notwithstanding the generalized rightward and downward response biases towards the hand's center of gravity: Coren \& Hoenig, 1972). This not only confirms the predictive perceptual bias for observed actions, but demonstrates that the perceptual bias reflects the natural trajectory of the arm when executing these actions forward and upward for reaches, and backward and downward for withdrawals - rather than a gross shift horizontally.

Secondly, the results replicate the intention-action prediction effect observed in the previous experiments. That is, the predictive perceptual biases were largest when the action corresponded to the prior intention statements: hands were judged to have disappeared nearest the object (left) for reaches after hearing "I'll take it", farthest from the object (right) for withdrawals after hearing "I'll leave it", as if the perceptual bias caused by the kinematics of the action were further exaggerated by the intention. These results validate the use of the touch screen to investigate how prior knowledge of an actor's intention elicits a predictive bias in the perception of their actions, and demonstrates the robustness of the effect across different methodologies.

Finally, and most importantly, Experiment 3 again showed, as Experiment 1, that if the intention reliably predicted the action, the extent to which it exaggerated the predictive perceptual bias was larger than if the intention was counter-predictive of the action. These results therefore confirm that the weighting with which different cues affect an action's perceptual representation are adjusted if explicit information about the cue-action relationships is available and this information is supported by the actual perceptual input. 


\section{General Discussion}

Across three experiments, we tested whether others statements about their intentions biases perceptual judgments of their actions in predictive manner, and whether these biases depend on the strength of the relationship between statement and subsequent action (predictive or counterpredictive). We relied on the phenomenon that the perception of moving stimuli is biased forward in time to a position that would have been seen if the movement had continued along the trajectory (Freyd \& Finke, 1984, Hubbard, 2005). Indeed, in the current study, a reach for an object was reported to be nearer to the object than its actual position, and a withdrawal from the object was perceived to be farther away. Importantly, replicating our prior work (Hudson et al, 2016 ${ }^{\mathrm{ab}}$ ), these action perception biases incorporated knowledge of the actor's goals: they increased when the actor revealed a congruent goal prior to acting, such that hands were reported to have disappeared even closer to the goal object if the statement implied the goal to pick it up, and further away when implying a withdrawal. These findings are in line with the idea that goals attributed to others' drive predictions of their actions, which in turn bias subsequent perceptual processes in the expected direction (Hudson et al, 2016 ${ }^{\mathrm{ab}}$ ).

Importantly, the results show for the first time that the extent to which intentional information drove action prediction is directly linked to the likelihood that the actor would actually do as they said. In other words, the automatic intention-action prediction effect observed by Hudson et al $\left(2016^{\mathrm{ab}}\right)$ can be "turned up or down" by controlling the predictive relationship between intention statement and action. This principle was demonstrated even though participants were not required to monitor the intention-action relationship and, in fact, the action statements were completely irrelevant to the participants' only task of accurately reporting the hand's disappearance point. Moreover, the effect was evident both between and within subjects, and with two different methodologies, suggesting that any prior associations made between the intention and action that influence perception can be rapidly revised in light of new information (see van Elk, van Schie, \& 
Bekkering, 2009 for similar results relating to action execution). The results therefore support the notion that the modulation of the predictive perceptual bias largely reflects the role of the intentional cues in predicting others' subsequent actions, and not, for example, embodied effects of the words that make up the intention statements ('I'll take it" or "I'll leave it'). The finding is therefore consistent with our observation (Hudson et al., 2016 ${ }^{\mathrm{b}}$ ) that the precise statements that the actors make, and the meaning is associated to them, is not relevant, as long as they can be reliably interpreted as predictors of their actions. The intention-action prediction effects were elicited equally well by terms referring to the actor's goals ("take it/leave it"), terms referring to termination or initiation of a movement ("Stop/Go") or by terms referring to movement direction from the actor's perspective ("forward/backward").

A striking finding was that it was not enough for participants to simply experience the statistical contingencies of intention statements and subsequent actions for the predictive biases to be adjusted, and nor was it sufficient for participants to merely be informed that the actor would most likely do (or not do) as they had said. The predictive role of the intention statements was only adjusted when participants were both given explicit information of the relationship and when this knowledge was supported by real world experience. This finding is in line with the proposal of predictive processing models that revisions in expected precision are relatively resource intensive: they require tracking and integrating of prediction errors across separate events in time, such that the observed relationship can be matched against the probability distribution expected from prior experience, and adjustments are not made after single violations ("outliers"), but only when predictions are consistently false (Hohwy, 2013). Such monitoring may therefore be only undertaken when prompted by higher-level information about the precision of the intention-action link, which can then be confirmed or rejected by actual perceptual experience (Friston \& Kiebel, 2009; Hohwy, 2013). 
This finding is remarkably similar to recent work in associative learning. While associative learning has classically been linked to highly automatic mechanisms that proceed outside awareness, this conclusion is increasingly doubted, on both methodological and theoretical grounds (e.g., Shanks \& John, 1994; Mitchell, De Houwer \& Lovibond, 2009). Instead, it has been argued that instructions have played a major (but under-acknowledged) role in directing participants to the relevant stimulus relationships and encouraging updating or revising the relevant internal models (for an early argument see, Alloy \& Tabachnik, 1984). Similar to here, more recent studies show that even classical measures of association learning, such as Pavlovian eye-blink conditioning or skin conductance responses in fear conditioning, crucially depend on participants being instructed about - or becoming aware of - the relevant contingencies; otherwise, learning remains essentially flat (e.g., Weidemann, Satkunarajah, \& Lovibond, 2016; Mertens \& De Houwer, 2016). Similar to our findings then, these studies outside a social learning context support the proposal of predictive coding models that revisions of the weighting of predictive influence is itself not just a bottom-up driven process, but one that is very much under top-down control and guided by prior information about the relevant relationships (Hohwy, 2013; Alloy \& Tabachnik, 1984).

In everyday life, where such prior information may be lacking, monitoring and updating of predictive relationships may be triggered in a different manner. The main difference to the current experimental situation is that others' intention statements are not task-irrelevant. Instead, we typically have a vested interest in the people we interact with and the consistency between their statements and their behavior is relevant for both judgments about them and actions with them, supporting anticipatory action planning and avoidance of errors, particularly in joint action situations (e.g., Sebanz, Bekkering, \& Knoblich, 2006). Prior research in the non-social domain suggests that it may be precisely such costly errors in judg ment or action that result from inadequate prediction, which trigger revisions of previously established relationships (Dale et al., 2012; Duran \& Dale, 2009; Marcus et al., 2006). Repeated failure of such "behavioral wagers" 
(Dale et al., 2012) may therefore be the relevant signal that makes predictive relationships tasks relevant and which triggers the required integration and monitoring processes, allowing previously established relationships to be adjusted in the light of new information.

An additional notable finding was that, in Experiments 1 and 3, the effect of a counterpredictive relationship between intention and action served only to reduce - but not reverse - the intention-action prediction effect. Indeed, even when the actor typically acted opposite to their stated intention, numerically the effect of the intention statements was in the same direction as when his actions were congruent with it. This shows firstly, that the predictive influence of stated intentions on action perception, established through long time experience with others' behavior, is automatic and difficult to eradicate entirely, persisting even in the light of conflicting explicit information or statistical evidence. Secondly, it suggests that the intention-action prediction effect was not due to a low level association between intention and action that could, in principle, be achieved with any predictive stimulus. If this were so, then a counter-predictive congruency effect would have been observed for the counter-predictive groups, with the predictive perceptual bias larger for actions that were incongruent with the intention than those that were congruent. However, this was not the case, suggesting that the effect of prior knowledge of an actor's intentions relies very much on the actions that such intentions typically bring about, and the up- and downregulation of these links in the light of congruent or incongruent experience.

Together, our findings are consistent with predictive coding models in the non-social domain that assume that expectations of what is likely to occur are organized hierarchically and integrated into a Bayesian model of the most likely perceptual input (Bubic, von Cramon \& Schubotz, 2010; Clark, 2013; den Ouden, Kok, \& De Lange, 2012; Friston \& Kiebel, 2009; Markov \& Kennedy, 2013). These predictions cascade downstream where incoming sensory information is weighted according to the prediction, biasing the perception of correctly predicted stimuli, and highlighting errors more easily. In social interactions, what we can glean of someone's goals and 
intentions may therefore enable a prediction of the actions that will most likely achieve their goals (Bach et al., 2014; Csibra, 2007; Hudson et al., 2016 ${ }^{\text {ab }}$ Keysers \& Gazzola, 2014; Kilner, 2011; Kilner, Friston \& Frith, $2007^{\mathrm{ab}}$ ), which then becomes integrated with other predictive information sources (e.g., eye movements: Hudson et al., 2009; 2011; 2012; prior trajectories: Kessler, et al., 2010; objects: Bach, Bayliss \& Tipper, 2011; Nicholson, Roser \& Bach, 2017).

Such a hierarchical integration can be seen in the current experiments. Firstly, on the most general level, perceptual displacement in the direction of motion was observed in all conditions of Experiments 1 and 2, irrespective of the prior intention or its predictability. Therefore, this general perceptual forward bias was driven by immediately available motion information, which is the most reliable predictor of the future position. This can be seen especially in Experiment 3, in which the hand was equally likely to reach or withdraw from the object irrespective of how well the intentional information predicted it. As a result, the effect of action on responses remained the same between conditions. Secondly, in all experiments, the maximal perceptual shift was observed when the motion reflected the previously stated goal of the actor, showing that top-down information about others' intention is integrated into one's predictions of forthcoming actions. Finally, the expected congruency of action and intention once it was observed increased the precision of the predicted future location. In contrast, where the actor was most likely to do the opposite, the precision of the predicted action, even if it was congruent with the intention, was weaker, and the sensory weighting and consequent predictive perceptual bias were reduced, reflecting only the immediately preceding motion information. This therefore shows that the predictive role of each cue can itself be adjusted if it fails to provide reliable predictions in the given context, such that is used less to predict others' actions, after the validity of the predictive intention cues has been monitored across events (Hohwy, 2013).

The application of hierarchical predictive models to social perception has the potential to reconcile a range of findings. Humans are acutely sensitive to cues that convey the goals and 
intentions of people (eye gaze, emotional expression) from the bare minimum of information (their face or gait). We are also keenly aware of the location and type of objects that could be acted upon (Bach, Nicholson, Hudson, 2014; Creem-Regehr, Gagnon, Geuss \& Stefanucci, 2013), to the extent that our perception of other people's eye gaze is drawn to these objects (Joyce et al., 2016; Lobmaier, Fischer \& Schwaninger, 2006). All of these cues can be combined to increase the precision of our predictions of how others will behave (Becchio, Bertone \& Castiello, 2008; Hunnius \& Bekkering, 2010). In line with such models, our data suggest that such predictions exert a top-down influence on the perceptual representation of others' subsequent behavior (e.g. Kessler et al., 2010), such that own actions can be anticipated and guided by these anticipated future states rather than what is present right now (i.e. Hubbard, 2006). Indeed, when acting with others we seem to be implicitly aware of our partners' need for reliability and we make our actions more predictable to facilitate such coordination (Vesper, Robrect, van der Wel, Knoblich, \& Sebanz, 2011).

The present findings of biased perceptual reports of actions is also consistent with perceptual distortions of the distal consequences of others actions. During action execution, we predict how our actions will alter the environment, and these desired changes determine how we select an action in the first place (Hommel, Müsseler, Aschersleben \& Prinz, 2001). The resulting perception of these action effects is often attenuated and temporally bound to our actions, so that perceptual reports of timing and stimulus intensity change when they are predicted by one's own actions (Haggard, Clark \& Kalogeras, 2002; Hughes, Desantis \& Waszak, 2013). The same perceptual biases are evident when we observe the others' actions, suggesting that similar perceptual mechanisms are used to predict the consequences of their behavior (Poonian \& Cunnington, 2013; Sato, 2008; Wohlschläger, Engbert \& Haggard, 2003; Wohlschläger, Haggard, Gesierich \& Prinz, 2003). While our study agrees with the reported changes to perceptual representations to others' actions, it differs in that these changes did not affect mere timing or intensity judgments of subsequent abstract stimuli (the actions' distal effects), but that predictions 
can already alter how an action's kinematics and trajectory are represented (its proximal effects), and that these influences reflect the goals attributed to them.

Thirdly, our results especially speak to recent suggestions that people adapt their expectations to suit specific individuals and situations. The predictability of an individual's behavior with respect to different objects enables us to attribute likes and dislikes to others and predict their actions on the basis of this knowledge (Joyce et al., 2016; Schenke et al., 2016), and we tend to trust those whose behavior is predictable and reliable (Bayliss \& Tipper, 2006), and who have a positive disposition towards us (Hudson, Nijboer \& Jellema, 2012). Our data suggest that one way how people achieve this adjustment is not only by generating novel internal models that describe these individual's behavior in different situations (Joyce et al., 2016; Schenke et al., 2016), but by up- or down- weighting the predictive influences of the cues other individuals give away, which have been established by prior experience. As a consequence, the perception of predictable people's actions will be strengthened by various prior cues that they have given away, allowing robust planning of own actions. In contrast, for less predictable people, the bottom-up biological motion input needs to be weighted more heavily, leading to impairments in coordination and action understanding.

Finally, predictive processes may play a prominent role in the evolution and development of social cognition. Infants as young as 1 year of age, as well as non-human primates, interpret the behavior of others to be purposeful and goal directed (Baillargeon, Scott \& Bian, 2016; Csibra \& Gergely, 2007; Gergely \& Csibra, 2003). As a result they expect others to act in the most efficient and rational way possible, and show surprise to actions that are unnecessarily convoluted or impractical (Rochat, Serra, Fadiga \& Gallese, 2008; Vivanti et al, 2011). The need to account for such unexpected behavior requires that the simple relations between the action, end goal, and environment be abstracted to mentalistic states of intentions, desires, and beliefs, providing a greater insight into the minds of others beyond that which is immediately perceivable, and forming 
the essence of human social cognition. Interruptions of this developmental trajectory may explain some of the deficits exhibited by those with autism and schizophrenia (Fletcher \& Frith, 2009; Van de Curys, Evers, Van der Hallen, Van Eylen, Boets, de-Wit \& Wagemans, 2014). An inability to modify or update the precision of their predictions about how the environment will change may go some way to explain the pervasive and wide ranging cognitive and perceptual deficits they exhibit, of which social interactions would be particularly susceptible due to the especially ambiguous link between other's behavior and the goals and intentions that cause them. 


\section{References}

Alloy, L. B., \& Tabachnik, N. (1984). Assessment of covariation by humans and animals: The joint influence of prior expectations and current situational information. Psychological Review, 91, 112-149. doi:10.1037//0033-295X.91.1.112

Bach, P., Bayliss, A. P., \& Tipper, S. P. (2011). The predictive mirror: interactions of mirror and affordance processes during action observation. Psychonomic Bulletin \& Review, 18(1), 171-176. doi:10.3758/s13423-010-0029-x.

Bach, P., Hudson, M., \& Nicholson, T. (2017). Perceptual Simulation. In preparation.

Bach, P., Knoblich, G., Gunter, T., C., Friederici, A., D., \& Prinz, W. (2005). Action comprehension: deriving spatial and functional relations. Journal of Experimental Psychology: Human Perception and Performance, 31, 465-79. doi:10.1037/00961523.31 .3 .465

Bach, P., Nicholson, T., \& Hudson, M. (2014). The affordance-matching hypothesis: How objects guide action understanding and prediction. Frontiers in Human Neuroscience. 8, 254. doi:10.3389/fnhum.2014.00254.

Bach, P. \& Schenke, K. (in press). Predictive social perception: towards a unifying framework from action observation to person knowledge. Social and Personality Psychology Compass.

Baillargeon, R., Scott, R. M., \& Bian, L. (2016). Psychological reasoning in infancy. Annual Review of Psychology, 67, 159-186. doi:10.1146/annurev-psych-010213-115033

Bayliss, A.P., \& Tipper, S.P. (2006). Predictive gaze and personality judgments: Should eye trust you? Psychological Science, 17, 514-520. doi:10.1111/j.1467-9280.2006.01737.x

Becchio, C., Bertone, C., \& Castiello, U. (2008). How the gaze of others influences object processing. Trends in Cognitive Sciences, 12, 254-258. doi:10.1016/j.tics.2008.04.005

Beersma, B. \& Van Kleef, G.A. (2012). Why people gossip: An empirical analysis of social motives, antecedents, and consequences. Journal of Applied Social Psychology, 42, 26402670. doi:10.1111/j.1559-1816.2012.00956.x 
Brown, E.C., \& Brune, M. (2012). The role of prediction in social neuroscience. Frontiers in Human Neuroscience, 6, article 147, 1-19. doi:10.3389/fnhum.2012.00147

Bubic, A., von Cramon, D.Y., \& Schubotz, R.I. (2010). Prediction, cognition and the brain. Frontiers in Human Neuroscience, 4, article 25. doi:10.3389/fnhum.2010.00025

Chatterjee, S.H., Freyd., J.J., \& Shiffrar, M. (1996). Configural processing in the perception of apparent biological motion. Journal of Experimental Psychology: Human Perception and Performance, 22, 916-929. doi:10.1037/0096-1523.22.4.916

Clark, A. (2013). Whatever next? Predictive brains, situated agents, and the future of cognitive science. Behavioral and Brain Sciences, 36, 1-73. doi:10.1017/S0140525X12000477

Coren, S. \& Hoenig, P. (1972). Effect of non-target stimuli upon length of voluntary saccades. Perceptual and Motor Skills, 34, 499-508. doi:10.2466/pms.1972.34.2.499

Creem-Regehr, S. H., Gagnon, K. T., Geuss, M. N., \& Stefanucci, J. K. (2013). Relating spatial perspective taking to the perception of other's affordances: providing a foundation for predicting the future behaviour of others. Frontiers in Human Neuroscience, 7, 596. doi:10.3389/fnhum.2013.00596

Csibra, G. (2007). Action mirroring and action interpretation: An alternative account. In: P. Haggard, Y. Rosetti, \& M. Kawato (Eds.), Sensorimotor Foundations of Higher Cognition. Attention and Performance XXII (pp. 435-459). Oxford: Oxford University Press. doi:10.1093/acprof:oso/9780199231447.003.0020

Csibra, G., \& Gergely, G. (2007). Obsessed with goals: Functions and mechanisms of teleological interpretation of actions in humans. Acta Psychologica, 124, 60-78. doi:10.1016/j.actpsy.2006.09.007

Dale, R., Duran, N.D., \& Morehead, J.R. (2012). Prediction during statistical learning, and implications for the implicit/explicit divide. Advances in Cognitive Psychology, 8, 196-209. doi:10.2478/v10053-008-0115-z 
Den Ouden, H.E.M., Kok, P., \& De Lange, F.P. (2012). How prediction errors shape perception, attention, and motivation. Frontiers in Psychology, 3, 1-12. doi:10.3389/fpsyg.2012.00548

Dennett, D. C. (1987). The intentional stance. Cambridge, MA: MIT Press.

Dils, A.T., \& Boroditsky, L. (2010). A Visual Motion Aftereffect from Motion Language. Proceedings of the National Academy of Sciences, 107, 16396-16400. doi:10.1073/pnas.1009438107

Duran, N.D., \& Dale, R. (2009). Anticipatory arm placement in the statistical learning of position sequences. In Proceedings of the 31st Annual Meeting of the Cognitive Science Society (pp. 893- 898). Amsterdam: Cognitive Science Society.

Fletcher, P. C., \& Frith, C. D. (2009). Perceiving is believing: A Bayesian approach to explaining the positive symptoms of schizophrenia. Nature Reviews Neuroscience, 10, 48-58. doi:10.1038/nrn2536

Freyd, J. J., \& Finke, R. A. (1984). Representational momentum. Journal of Experimental Psychology: Learning, Memory, and Cognition, 10, 126-132. doi:10.1037/02787393.10.1.126

Freyd, J. J., \& Johnson, J. Q. (1987). Probing the time course of representational momentum. Journal of Experimental Psychology: Learning, Memory, and Cognition, 13, 259-269. doi:10.1037/0278-7393.13.2.259

Friston, K.J., \& Kiebel, S. (2009). Cortical circuits for perceptual inference. Neural Networks, 22, 1093-1104. doi:10.1016/j.neunet.2009.07.023.

Gergely, G., \& Csibra, G. (2003). Teleological reasoning in infancy: The nave theory of rational action. Trends in Cognitive Sciences, 7, 287-292. doi:10.1016/S1364-6613(03)00128-1

Goldreich, D. (2007). A Bayesian perceptual model replicates the cutaneous rabbit and other tactile spatiotemporal illusions. PLOS one, 3, e333. doi:10.1371/journal.pone.0000333

Graf, M., Reitzner, B., Corves, C., Casile, A., Giese, M., \& Prinz, W. (2007). Predicting point-light 
actions in real-time. Neuroimage, 36 (Supplement 2), T22-T32.

doi:10.1016/j.neuroimage.2007.03.017

Haggard, P., Clark, S., \& Kalogeras, J. (2002). Voluntary action and conscious awareness. Nature Neuroscience, 5, 382-385. doi:10.1038/nn827

Hommel, B., Müsseler, J., Aschersleben, G., \& Prinz, W. (2001). The Theory of Event Coding (TEC): A framework for perception and action planning. Behavioral and Brain Sciences, 24, 878-937. doi:10.1017/S0140525X01000103

Hohwy, J. (2013). The Predictive Mind. Oxford, UK: Oxford University Press.

Hubbard, T. L. (2006). Bridging the gap: Possible roles and contributions of representational momentum. Psicologica, 27, 1-34.

Hubbard, T. L. (2005). Representational momentum and related displacements in spatial memory: A review of the findings. Psychonomic Bulletin \& Review, 12, 822-851. doi:10.3758/BF03196775

Hubbard, T. L. (2013). Do the flash-lag effect and representational momentum involve similar extrapolations? Frontiers in Psychology, 4, article 290. doi:10.3389/fpsyg.2013.00290.

Hubbard, T. L. (2014). Forms of momentum across space: Representational, operational, and attentional. Psychonomic Bulletin \& Review, 21, 1371-1403. doi:10.3758/s13423-014-06243

Hudson, M., Burnett, H.G., \& Jellema, T. (2012). Anticipation of intentional actions in highfunctioning autism. Journal of Autism \& Developmental Disorders, 42, 1684-1693. doi:10.1007/s10803-011-1410-y

Hudson, M., Hong-Liu, C., \& Jellema, T. (2009). Anticipating intentional actions: The effect of eye gaze direction on the judgment of head rotation. Cognition, 112, 423-434. doi:10.1016/j.cognition.2009.06.011

Hudson, M., \& Jellema, T. (2011). Resolving ambiguous behavioural intentions by means of 
involuntary prioritisation of gaze processing. Emotion, 11, 681-686. doi:10.1037/a0023264

Hudson, M., Nicholson, T., Ellis, R., \& Bach, P. (2016a). I see what you say: Prior knowledge of other's goals automatically biases the perception of their actions. Cognition, 146, 245-250. doi:10.1016/j.cognition.2015.09.021

Hudson, M., Nicholson, T., Simpson, W.A., Ellis, R., \& Bach, P. (2016b). One step ahead: The perceived kinematics of others' actions are biased towards expected goals. Journal of Experimental Psychology: General, 145, 1-7. doi:10.1037/xge0000126

Hudson, M., Nijboer, T., \& Jellema, T. (2012). Implicit learning of social information and its relation to autistic traits. Journal of Autism \& Developmental Disorders, 42, 2534-2545. doi:10.1007/s10803-012-1510-3

Hughes, G., Desantis, A., \& Waszak, F. (2013). Mechanisms of intentional binding and sensory attenuation: The role of temporal prediction, temporal control, identity prediction, and motor prediction. Psychological Bulletin, 139, 133-151. doi:10.1037/a0028566

Hunnius, S., and Bekkering, H. (2010). The early development of object knowledge: a Study of infants' visual anticipations during action observation. Developmental Psychology, 46, 446-454. doi:10.1037/a0016543

Jarraya, M., Amorim, M. A., \& Bardy, B. G. (2005). Optical flow and viewpoint change modulate the perception and memorization of complex motion. Perception \& Psychophysics, 67, 951961. doi:10.3758/BF03193622

Joyce, K., Schenke, K., Bayliss, A.P., \& Bach, P. (2016). Looking ahead: Anticipatory cueing of attention to objects others will look at. Cognitive Neuroscience, 7, 74-81, doi:10.1080/17588928.2015.1053443

Kessler, K., Gordon, L., Cessford, K., \& Lages, M. (2010). Characteristics of motor resonance predict the pattern of flash-lag effects for biological motion. PLOS One, 5, e8258. doi:10.1371/journal.pone.0008258 
Keysers, C., \& Gazzola, V. (2014). Hebbian learning and predictive mirror neurons for actions, sensations and emotions. Philosophical Transactions of the Royal Society of London. Series B, Biological Sciences, 369, 20130175. doi:10.1098/rstb.2013.0175.

Kilner, J. (2011). More than one pathway to action understanding. Trends in Cognitive Sciences, 15, 352-357. doi:10.1016/j.tics.2011.06.005

Kilner, J.M., Friston, K.J., \& Frith, C.D. (2007a). Predictive coding: An account of the mirror neuron system. Cognitive Processing, 8, 159-166. doi:10.1007/s10339-007-0170-2

Kilner, J.M., Friston, K.J., \& Frith, C.D. (2007b). The mirror-neuron system: A Bayesian perspective. NeuroReport, 18, 619-623. doi:10.1097/WNR.0b013e3281139ed0

Koster-Hale, J. \& Saxe, R. (2013). Theory of Mind: A neural prediction problem. Neuron, 79, 836848. doi:10.1016/j.neuron.2013.08.020

Kourtzi, Z., \& Shiffrar, M. (1997). One-shot invariance in a moving world. Psychological Science, 8, 461-466. doi:10.1111/j.1467-9280.1997.tb00462.x

Kourtzi, Z., \& Shiffrar, M. (1999). Dynamic representations of human body movement. Perception, 28, 49-62. doi:10.1068/p2870

Lafer-Sousa, R., Hermann, K.L., \& Conway, B.R. (2015). Striking individual differences in color perception uncovered by 'the dress' photograph. Current Biology, 25, R523-R548. doi:10.1016/j.cub.2015.04.053

Lobmaier, J. S., Fischer, M. H., \& Schwaninger, A. (2006). Objects capture perceived gaze direction. Experimental Psychology, 53, 117-122. doi:10.1027/1618-3169.53.2.117

Manera, V., Schouten, B., Verfaillie, K., \& Becchio, C. (2013). Time will show: Real time predictions during interpersonal action perception. PLOS One, 8, e54949. doi:10.1371/journal.pone.0054949

Marcus, D.J., Karatekin, C., \& Markiewicz, S. (2006). Oculomotor evidence of sequence learning 
on the serial reaction time task. Memory \& Cognition, 34, 420-432. doi:10.3758/BF03193419

Markov, N. T., \& Kennedy, H. (2013). The importance of being hierarchical. Current opinion in Neurobiology, 23, 1-8. doi:10.1016/j.conb.2012.12.008

Mertens, G., \& De Houwer, J. (2016). Potentiation of the startle reflex is in line with contingency reversal instructions rather than the conditioning history. Biological Psychology, 113, 91-99. doi:10.1016/j.biopsycho.2015.11.014

Meteyard, L., Bahrami, B., \& Vigliocco, G. (2007). Motion Detection and Motion Verbs: Language Affects Low-Level Visual Perception. Psychological Science, 18, 1007-1013. doi:10.1111/j.1467-9280.2007.02016.x

Mitchell, C. J., De Houwer, J., \& Lovibond, P. F. (2009). The propositional nature of human associative learning. Behavioral and Brain Sciences, 32, 183-198. doi:10.1017/S0140525X09000855

Munger, M. P., \& Minchew, J. H. (2002). Parallels between remembering and predicting an object's location. Visual Cognition, 9, 177-194. doi:10.1080/13506280143000386

Neri, P., Luu, J.Y., \& Levi, D.M. (2006). Meaningful interactions can enhance visual discrimination of human agents. Nature Neuroscience, 9, 1186-1192. doi:10.1038/nn1759

Nicholson, T., Roser, M., \& Bach, P. (2017). Understanding the Goals of Everyday Instrumental Actions Is Primarily Linked to Object, Not Motor-Kinematic, Information: Evidence from fMRI. PLOS ONE, 12(1), e0169700. doi:10.1371/journal.pone.0169700

Nijhawan, R. (2002). Neural delays, visual motion and the flash-lag effect. Trends in Cognitive Sciences, 6, 387-424. doi:10.1016/S1364-6613(02)01963-0

Orgs, G., Bestmann, S., Schuur, F., \& Haggard, P. (2011). From body to biological motion: Apparent velocity of human movement biases subjective time. Psychological Science, 22, 712-717. doi:10.1177/0956797611406446 
Parkinson, J., Springer, A., \& Prinz, W. (2012). Before, during and after you disappear: Aspects of timing and dynamic updating of the real-time action simulation of human motions. Psychological Research, 76, 421-433. doi:10.1007/s00426-012-0422-3

Poonian, S.K., \& Cunnington, R. (2013). Intentional binding in self-made and observed actions. Experimental Brain Research, 229, 419-427. doi:10.1007/s00221-013-3505-5

Pozzo, T., Papaxanthis, C., Petit, J.L., Schweighofer, N., \& Stucchi, N. (2006). Kinematic features of movement tunes perception and action coupling. Behavioural Brain Research, 169, 7582. doi:10.1016/j.bbr.2005.12.005

Rochat, M. J., Serra, E., Fadiga, L., \& Gallese, V. (2008). The evolution of social cognition: goal familiarity shapes monkeys' action understanding. Current Biology, 18, 227-232. doi:10.1016/j.cub.2007.12.021

Sarrazin, J-C., Giraudo, M-D., Pailhous, J., \& Bootsma, R.J. (2004). Dynamics of balancing space and time in memory: Tau and Kappa effects revisted. Journal of Experimental Psychology: Human Perception and Performance, 30, 411-430. doi:10.1037/0096-1523.30.3.411

Sato, A. (2008). Action observation modulates auditory perception of the consequences of others' actions. Consciousness and Cognition, 17, 1219-1227. doi:10.1016/j.concog.2008.01.003

Saunier, G., Papaxanthis, C., Vargas., \& Pozzo, T. (2008). Inference of complex human motion requires internal models of action: Behavioural evidence. Experimental Brain Research, 185, 399-409. doi:10.1007/s00221-007-1162-2

Saxe, R., Tenenbaum, J. B., \& Carey, S. (2005). Secret agents: inferences about hidden causes by 10- and 12-month-old infants. Psychological Science, 16, 995-1001. doi:10.1111/j.14679280.2005.01649.x

Schenke, K.C., Wyer, N.A., \& Bach, P. (2016). The Things You Do: Internal Models of Others' Expected Behaviour Guide Action Observation. PloS One, 11, doi:10.1371/journal.pone.0158910. 
Sebanz, N., Bekkering, H. \& Knoblich, G. (2006). Joint action: Bodies and minds moving together. Trends in Cognitive Sciences, 10, 70-76. doi: 10.1016/j.tics.2005.12.009

Shanks, D. R., \& John, M. F. S. (1994). Characteristics of dissociable human learning systems. Behavioral and Brain Sciences, 17, 367-395. doi:10.1017/S0140525X00035032

Shiffrar, M., \& Freyd, J.J. (1990). Apparent motion of the human body. Psychological Science, 1, 257-264. doi:10.1111/j.1467-9280.1990.tb00210.x

Shiffrar, M., \& Freyd, J.J. (1993). Timing and apparent motion path choice with human body photographs. Psychological Science, 4, 379-384. doi:10.1111/j.1467-9280.1993.tb00585.x

Shiffrar, M., Lichtey, L., \& Chatterjee, S.H. (1997). The perception of biological motion across apertures. Perception and Psychophysics, 59, 51-59. doi:10.3758/BF03206847

Stone, J.V., Kerrigan, I.S., \& Porrill, J. (2009). Where is the light? Bayesian perceptual priors for lighting direction. Proceedings of the Royal Society B: Biological Sciences, 276, 1797-804. doi:10.1098/rspb.2008.1635

Teufel, C., Fletcher, P. C., \& Davis, G. (2010). Seeing other minds: Attributed mental states influence perception. Trends in Cognitive Sciences, 14, 376-382. doi:10.1016/j.tics.2010.05.005.

Thornton, I. M., \& Hayes, A. E. (2004). Anticipating action in complex scenes. Visual Cognition, 11, 341-370. doi:10.1080/13506280344000374

Van de Curys, S., Evers, K., Van der Hallen, R., Van Eylen, L., Boets, B., de-Wit, L., \& Wagemans, J. (2014). Precise minds in uncertain worlds: Predictive coding in autism. Psychological Review, 121, 649-675. doi:10.1037/a0037665

Van Elk, M., van Schie, H., \& Bekkering, H. (2009). Short-term action intentions overrule longterm semantic knowledge. Cognition, 111, 72-83. doi:10.1016/j.cognition.2008.12.002

Van Elk, M., van Schie, H., \& Bekkering, H. (2014). Action semantics: A unifying conceptual framework for the selective use of multimodal and modality-specific object knowledge. 
Physics of Life Reviews, 11, 220-250. doi:10.1016/j.plrev.2013.11.005

Vesper, C., Robrect, P. R. D. van der Wel, Knoblich, G., \& Sebanz, N. (2011). Making oneself predictable: Reduced temporal variability facilitates joint action coordination. Experimental Brain Research, 211, 517-530. doi:10.1007/s00221-011-2706-z

Vivanti, G., McCormick, C., Young, G.S., Abucayan, F., Hatt, N., Nadig, A., Ozonoff, S., \& Rogers, S.J. (2011). Intact and impaired mechanisms of action understanding in autism. Developmental Psychology, 47, 841-856. doi:10.1037/a0023105.

Weidemann, G., Satkunarajah, M., \& Lovibond, P. F. (2016). I think, therefore eyeblink: The importance of contingency awareness in conditioning. Psychological Science, 27, 467-475. doi:10.1177/0956797615625973

Wilson, M., Lancaster, J., \& Emmorey, K. (2010). Representational momentum for the human body: Awkwardness matters, experience does not. Cognition, 116, 242-250. doi:10.1016/j.cognition.2010.05.006.

Wohlschläger, A. (2000). Visual motion priming by invisible actions. Vision Research, 40, 925930. doi:10.1016/S0042-6989(99)00239-4

Wohlschläger, A., Engbert, K., \& Haggard, P. (2003). Intentionality as a constituting condition for the own self - and other selves. Consciousness and Cognition, 12, 708-716. doi:10.1016/S1053-8100(03)00083-7

Wohlschläger, A., Haggard, P., Gesierich, B., \& Prinz, W. (2003). The perceived onset time of self and other generated actions. Psychological Science, 14, 586-591. doi:10.1046/j.09567976.2003.psci_1469.x

Zaki, J. (2013). Cue integration: A common framework for social cognition and physical perception. Perspectives of Psychological Science, 8, 296-312. doi:10.1177/1745691613475454 